\title{
NON-ANALYTIC FUNCTIONS OF A COMPLEX VARIABLE
}

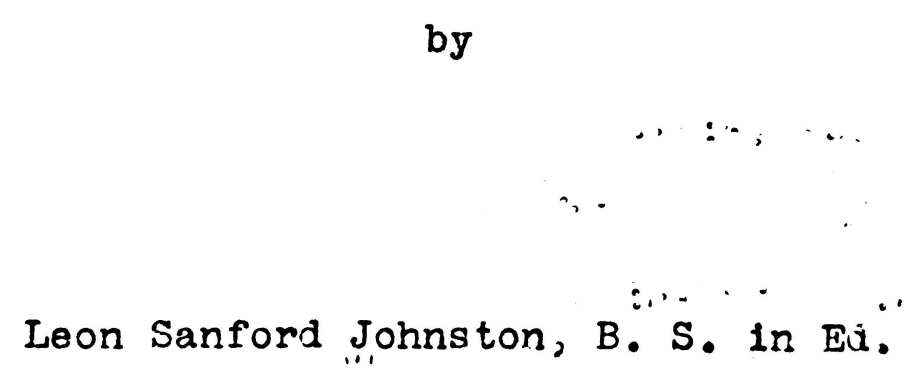

SUBMITTED IN PARTIAL FULFIILMENT OF THE REQUIREMENTS FOR THE DEGREE OF MASTER OF ARTS

in the

GRADUATE SCHOOL

of $\operatorname{tin} \theta$

UNIVERS ITY OF MISSOURI 
$X J 64$

NON-ANALYTIC,FUNCTIONS OF A

COMPLEX VARIABLE

INTRODUCTION.

It will be the purpose of this paper to discuss some of the properties of non-analytic functions of a complex variable, and to determine, if possible, some correspondences between the theory of analytic functions and the theory of non-analytic functions, if such a theory as the latter can be said to exist.

In the beginning, it is evident that the field of research in analytic functions is very limited, for it has been proved that the necessary and sufficient condition that a function be analytic is that the derivative shall exist. It is evident further that we shall be unable to conduct extensive researches in the similarity of the two classes of functions, since the theory of analytic functions depends so largely upon the existence of the derivative. It will be possible, however, to discover some properties of non-analyilc functions which are peculiar to such functions, and this paper will be also an attempt to discover and discuss some of these properties. The whole field of non-analytic functions is very large and difficult to classify, and it is precisely for the reason that the usual methods of analysis are inadequate, that investigations concerning them are not usually carried farther than they are.

While constant reference is made in this discussion to 
the theory of analytic functions, and some of the theorems stated therein are used and in some cases explained, yet it will presupposed that the reader is already acqua inted with the analytic theory, especially with the theory of Riemann Surfaces.

The avaliable material for reading on this subject is very limited, and reference is made in the thesis to two works only, and those on Calculus and Differential Equations. Reference has been made to Goursat's Course in llathematical Analysis, Volume I, (referred to as GoursatI) and to Murray's Differential Equations ( referred to as Murray). These works are described more completely in the Bibliography at the end of the thesis. 


\section{CHAPTER I}

A function $w=f(z)$, where $z=x+y 1$, is defined as analytic if the Cauchy-Riemann equations are satisfied; that is, w being expanded to the form $U(x, y)+V(x, y) i, U$ and $V$

being real, the partial differential equations $\frac{\partial U}{\partial x}=\frac{\partial V}{\partial y}, \frac{\partial V}{\partial x}=-\frac{\partial u}{\partial y}$ are satisfied. This is equivalent to saying that a function is analytic if its derivative exists, and conversely, if the function is analytic, the derivative must exist. We shall therefore defire as non-analytic a function of the form $w=U(x, y)+V(x, y) 1, U$ and $V$ being, as before, real, but not satisfying the Cauchy Riemann equations.

As has been stated before, the derivative of $w$, as last defined, does not exist; however, it has been shown that the functional determinant $\frac{D(U, V)}{D(X, Y)}$, in two variables, is analogous to the derivative in one variable. In the treatment of nonanalytic functions, therefore, the functional determinant, of Jacobian, will be used instead of the derivative in the earlier case.

In the analytic case, the branch points in the Rlemann surface were located by setting the derivative $d w / d z=0$, and solving for $z$. Now in the new case we shall have to set the Jacobian equal to zero, and solve for $x$ and $y$, in order to 10cate the branch points. Immediately one peculiar feature of the new class of functions presents itself. The derivative is the limit of the ratio of two infinitesimal lines, while the Jacobian is the limit of tro infinitesimal areas. ${ }^{1}$ The derivative, then, can vanish at points only, whlle here

Goursat, I, page 265, Art. 127. 
the Jacoblan may vanish at points or along entire lines or segments of jines. It is more common, indeed, for the Jacobian to vanish along entire lines than at points, and this feature is unique. Some very remarkable surfaces result from this property. Several such surfaces are discussed in this paper, and at least one general theorem arises from this propety. ${ }^{1}$

It can be shown that this method of locating the branch points or branch lines is identical with the nethod of the analytic case, where the derivative $\frac{d w}{d z}$ was used, and set equal to zero; for the Jacobian in the analytic case is $\frac{\partial u}{\partial x} \frac{\partial v}{\partial y}-\frac{\partial v}{\partial x} \frac{\partial u}{\partial y}=\left(\frac{\partial u}{\partial x}\right)^{2}+\left(\frac{\partial u}{\partial y}\right)^{2}=\left(\frac{\partial v}{\partial x}\right)^{2}+\left(\frac{\partial v}{\partial y}\right)^{2}=0$, which is satisfied only by $\frac{\partial u}{\partial x}=\frac{\partial u}{\partial y}=\frac{\partial v}{\partial x}=\frac{\partial v}{\partial y}=0$; but the derivative $\frac{d w}{d z}$, when expressed in terms of partial derivatives, is $\frac{\partial \alpha}{\partial x}+i \frac{\partial \alpha}{\partial y}=$ $\frac{\partial v}{\partial y}-i \frac{\partial v}{\partial x}$, and in order that the derivative be zero, all these partials must be zero, wilich is the condition for zero value of the Jacoblan. Hence the new method of locating the branch points is not really new, but is of much less restricted aplication than the older one.

Some explanation will be required for the term "branch Iir $\theta^{\prime}$. Me can not pass around the branch point, in the older case, without reaching a new value of the function. In the new case, we can not cross the Iine along which the Jacoblan vanishes without reaching a new value of the function. The term "branch line" is therefore justifiable by analogy with the older case of the branch point. Since the entireline

1. Chapter III. Page 43. 
can not be crossed, the branch line forces a fold in the surface, and the idea of "edge of regression" is suggested, which will be discussed in some detail later. ${ }^{1}$

The branch line is seen to differ very materially from the "cut" hsed in the analytic case to prevent circulation around a branch point; for these cuts were made in arbitrary positions, to suit the convenience of the occasion, while the branch 1 ine is determined by the conditions of the problem itself, and may not be changed in position, nor may it be crossed in any direction, as the cut freel permits. Just as the derivativemay vanish at more than one point, so the Jaooblanimay vanish at more than one point, or along more than one Iine, or at points and along lines. Examples will be discussed illustrating most of these cases, and methods of setting up functions with any desired branch points or lines will be shown.

We shall discuss some examples of analytic functions in Riemann Surfaces, to introduce the whole subject anew. The simplest example, perhaps, is the function $w=z^{2}$, where $z=x+y 1$. Setting the derivative $d v / d z=0$, we have $z=0$ as the only branch point, and that of the first order. This corresponds to the point $w=0$. Now we may cut the $W$ plane along. the $U$ axis to the right of the point $W=0$, which is equivalen't to cutting the $Z$ plane along the entire $X$ axis. This is shown by expanding $z^{2}$ to the form $x^{2}-y^{2}+2 x y 1$. Now in order for $V$ to be zero and $U$ greater than zero, as along this cut, we

Chapter III. Page 43. 
Figure 1.

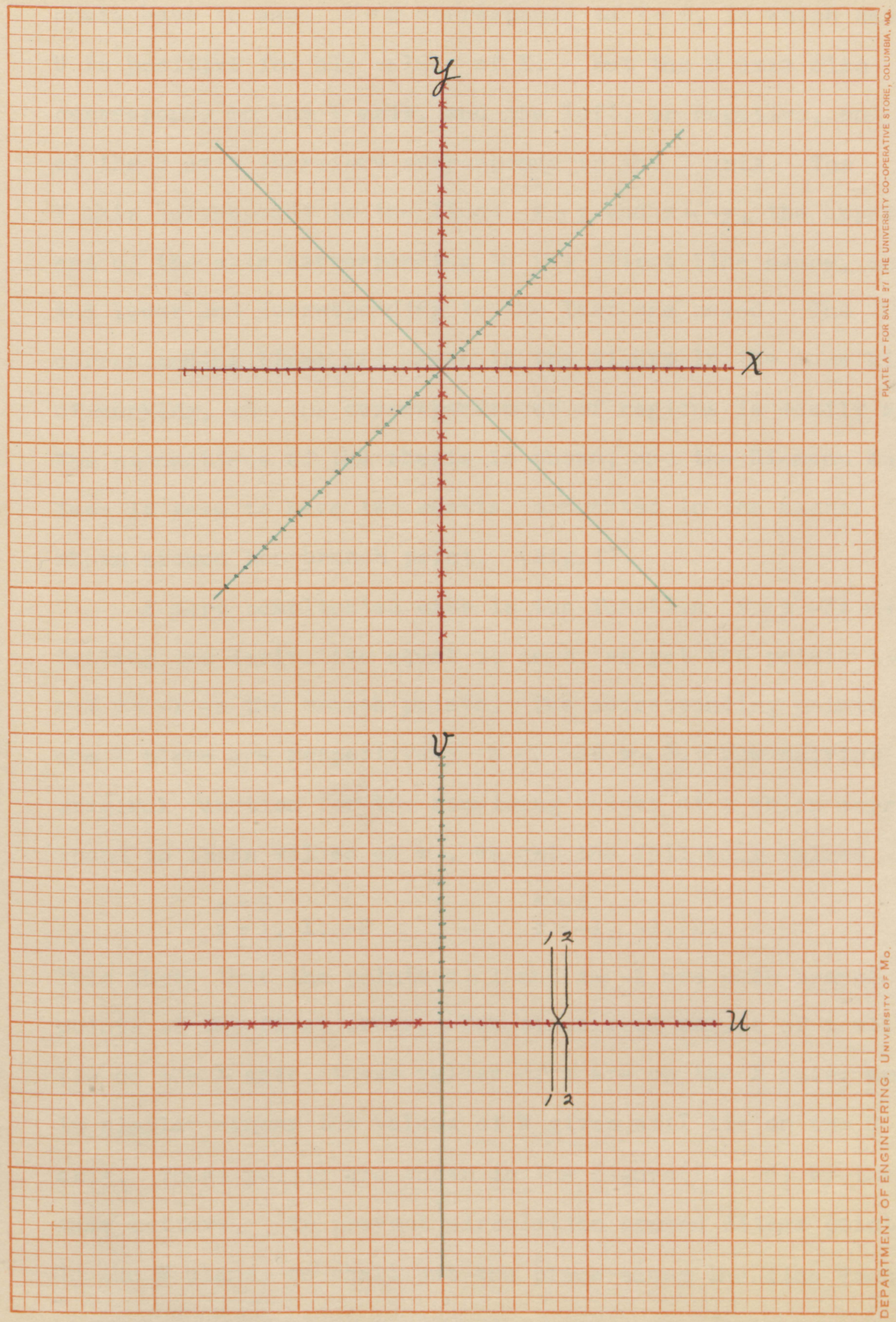


shall have to say. $V=0$ corresponds to $Y=0$, in which case $U$ is positive for all values of $X$. But the $U$ axis also corresponds to the $Y$ axis, and the left, or negative, end of the $U$ axis therefore corresponds to the $Y$ axis. The $V$ axis corresponds to the lines $x=y$ and $x=-y$, the upper end of the axis corresponding to the first line named, and the lower end corresponding to the last one named. The corresponding lines are drawn in the flgure in the same kinds of marking, and the correspondences of regions is easily seen. The connections of the sheets is shown, and the Rlemann surface is seen to have two sheets, connected in the manner shown around the branch point. (Fig.I)

A more complicated case is the function $w=z^{3}-3 z+4$. Setting the derivative equal to zero, we get the points $z=1$ and $z=-1$ as the branch points. The corresponding points in the $W$ plane are respectively $w=2$ and $w=6$. Now solving the original function for the remaining values of $z$ which correspond to these valges of $w$, we get the following values

$$
\begin{array}{ll}
w=2 & w=6 \\
z=1,1,-2 & z=-1,-1,2 .
\end{array}
$$

This shows tinat the points $z=1$ and $z=-1$ are each branch points of the first order. Now since real values of $z$ will lie on the $X$ axis, these values of $z$ are on the $X$ axis; and in similar manner the values of $\pi$ given will lie on the $U$ axis. These points are shown in the figure(Fig. 2) by the same letters. To prevent circulation around eitier point In the $\pi$ plane, we shall cut the plane along the line $V=0$, 
to the right from $w=6$, and to the left from $w=2$. To get the values of $U$ and $V$, we shall expand the original function, and set the real and imaginary parts equal to $U$ and $V i$ respectively; this gives $U=x^{3}-3 x y^{2}-3 x+4$, and $V=3 x^{2} y-y^{3}-3 y$. Now when $V=0$, as in the cuts described above, we have as the corresponding lines in the $Z$ plane the line $\mathrm{y}=0$ and the hyperbola $3 \mathrm{x}-\mathrm{y}-3=0$. When $\mathrm{z}>2$ and real, $w>6$ and real, and $w$ increases without limit as $z$ increases without limit. Therefore the part of the $U$ axis to the right of the point $U=6$ corresponds to the part of the $X$ axis to the right of the point $X=2$. In a similar manner it can be shown that the part of the U axis to the left of $U=2$ corresponds to the part of the $X$ axis to the left of -2. Now to determine the correspondences of the branches of the hyperbola, we have only to notice that the branch which includes the point $z=1$ must correspond to the line in the W plane which includes the corresponding point-this is the point $U=2$, as already pointed out. In like manner the other branch of the hyperbola must correspond to the line to the right of the point corresponding to $z=-1$ - that is, to the line which includes the point $U=6$. These lines are drawn in the same style of marking, and the correspondences can easily be determined. Finally, we can identify regions still more definitely if we draw the lines in the $\mathrm{z}$ planento the Iine $U$ 0. These Iines are drawn in the diagram in red ink. It will be noted that as we pass to the left from the point $U=2$, along the $U$ axis, the positive end of the $V$ axis is on 


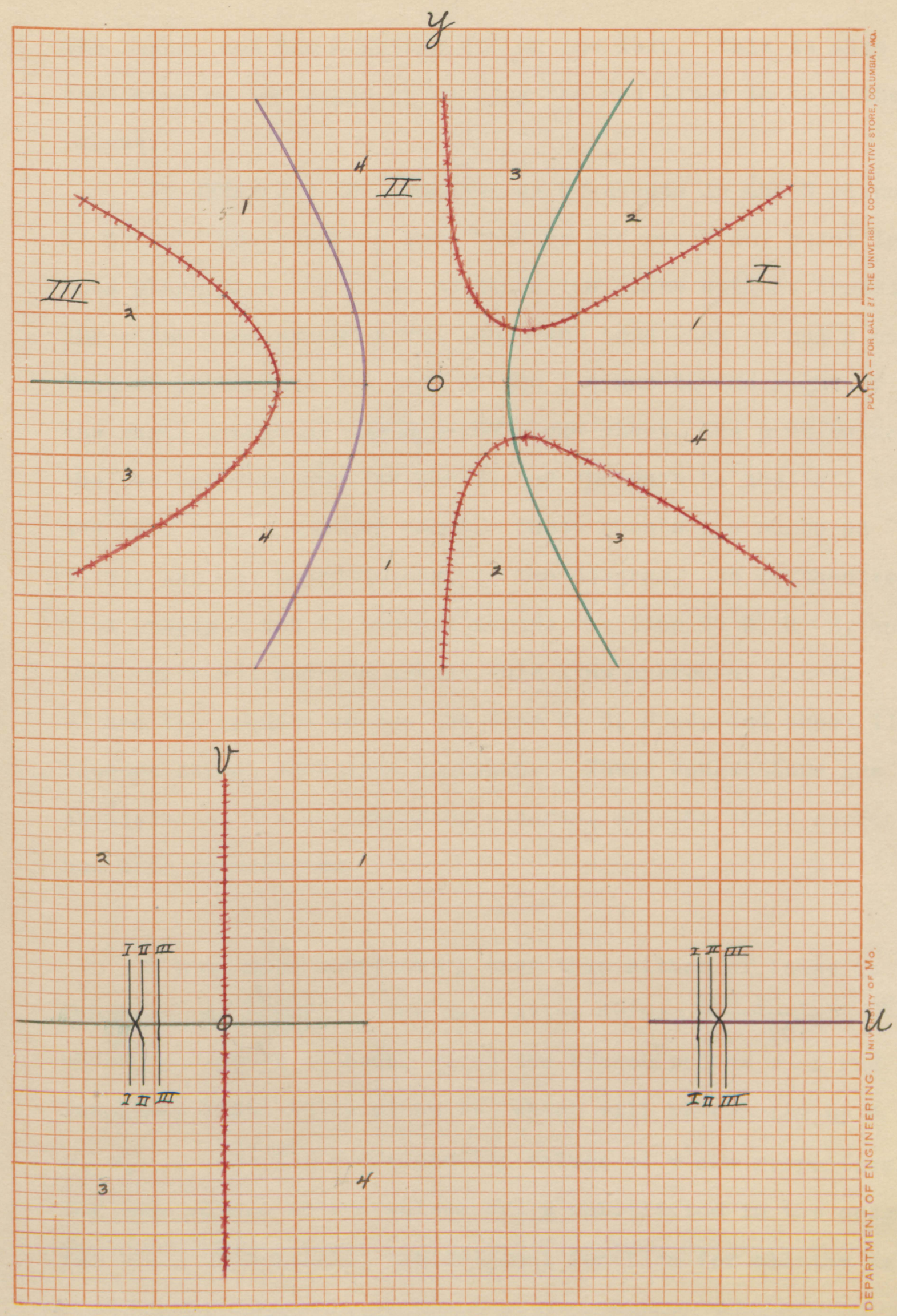


the right, and the negative end on the left. This is also Indicated by the style of marking the red lines in both the planes.

To determine the comnections of sheets in the $\$$ plane, it is only necessary to note that we can reach any point in this plane from any other point in the plane without crossing any other line except a red line, and this line is not a cut. Then we shall have the part of the $Z$ plane to the right of the right branch of the hyperbola one sheet, the part between the two branches another sheet, and the part to the left of the left branch another sheet. These sheets are numbered I, II, and III respectively, in the diagram. It is then seen that sheets I and II connect across the Iine $V=0, U<2$, and that sheet III extends across this Iine unbroken; sheets II and III connect across the line $V=0, U>6$; and sheet II extends across this line unbroken. These connections are indicated in the accompanying diagram, and the whole surface has been constructed.

A much more complicated case than either of these is the function $w=\frac{4 z^{5}+1}{5 z^{4}}$. The derivative $d w / d z=\frac{100 z^{8}-80 z^{8}-20 z^{3}}{25 z^{8}}$ which, set equal to zero, gives the branch points $z=1$ and the imaginary fifth roots of unity, which will be denoted by $\alpha_{1} \alpha_{2}$ $\alpha_{3} \alpha_{t}$, and the corresponding values of $w$ are respectively the same values as the values of $z$ substituted in the original function. Now solving the original function for all the values 
of $z$ which correspond to the values of $w$, we have, corresponding to $w=1$, the values $z=1,1$, and the roots of the equation $4 z^{3}+3 z^{2}+2 z+1=0$, which, by Horner's process are found to be $z=-0.606$ and $z=-0.072 \pm 0.6391$. For the values of $z$ corresponding to the other values of $w$, it is easily shown that the values of $z$ derived above are merely permuted at intervals of $\frac{2 \pi}{5}$, since the multipliers are respectively $\alpha_{1} \alpha_{2} \alpha_{3}$ and $\alpha_{4}$. The point $z=1$ is a branch point of the first order; hence the branches of the curve which pass through this point will make angles half as large as the corresponding curves though the point $w=1$. This remark applies also to the points $z=\alpha_{1} \alpha_{2} \alpha_{3} \alpha_{4}$ respectively.

The original function may be written $w=\frac{4}{5} z+\frac{1}{5} z^{-4}$. Changing to polar coordinates, we have

$$
\mathrm{U}+\mathrm{Vi}=\frac{4}{5} \mathrm{r}(\cos \theta+1 \sin \theta)+\frac{1}{5} \mathrm{r}^{4}[\cos (-4 \theta)+1 \sin (-4 \theta)]
$$

Separating the real and imaginary parts of this function, we have $U=\frac{4}{5} r \cos \theta+\frac{1}{5} r^{-4} \cos 4 \theta \quad V=\frac{4}{5} r \sin \theta-\frac{1}{5} r^{-4} \sin 4 \theta$. The $U$ axis is represented by setting to value of $V$ equal to zero, and solving the resulting equation. We have then the equation $\quad r^{5}=\frac{\sin 4 \theta}{4 \sin \theta}=\cos \theta \cos 2 \theta$, the factor $\sin \theta$ being removed. When the value sine is used in the original expression for $V, r$ may have any value, finite or infinite, and still satisft the relation for $V$ given. By assigning various values to $\theta$ in the last equation written, we can get the corresponding values of $r$. It is seen that when integral

$\theta$ is anyamultiple of $\frac{\pi}{4}$, the value of $r$ is zero; it is also seen that since $r^{5}$ must be positive, $\theta$ can assume only 
values between 0 and $\frac{\pi}{4}$, between $\frac{\pi}{2}$ and $\frac{3 \pi}{4}$, between $\frac{5 \pi}{4}$ and $\frac{3 \pi}{2}$, and between $\frac{7 \pi}{4}$ and $2 \pi$. The curve $\mathbf{r}^{5}=\cos \theta \cos 2 \theta$ is symmetrical with respect to the axis of origin, since $\cos \theta=\cos (-\theta)$ and $\cos 2 \theta=\cos (-2 \theta)$. The curve must then pass through the origin at angles of $\frac{\pi}{4}, \frac{\pi}{2}$, and $\frac{3 \pi}{4}$ with the original position of r. This alds materially in drawing the curve. By differentiation it can be shown that the curve must be vertical where $r=1$ and $\theta=0$.

From the original function, it is seen that when $w=0$, the value of $z$ is $\sqrt[5]{-\frac{1}{4}}$, which is represented by the point marked 0 , to the left of the origin on the $X$ axis (Fig. 3a). A : valuable check on the work so far is the fact that the values $\frac{\pi}{5}, \frac{3 \pi}{3}, \frac{5 \pi}{5}, \frac{2 \pi}{5}$ substituted in the relation $r^{5}=\cos \theta \cos 2 \theta$ give $r=5 \frac{1}{4}$, and these points are the same points as those located by setting the value of $w=0$, and permuting the corresponding point around the origin at intervals of $\frac{2 \pi}{5}$. From the original function $i t$ is also seen that $z=0$ gives an infinite value of $w$; therefore the origin in the $Z$ plane corresponds to the point at infinity in the $W$ plane. By differentiation we can find the maxiria of $r$; these are found at $0,112^{\circ}$; and $248^{\circ}$, the last two values being approxinate ( the exact angles are $\sin ^{-1} 0$ and $\cos ^{-1}\left( \pm \frac{1}{6} \sqrt{6}\right)$, but the plus sign in the latter expression can not be used, nor the value $\pi$ in the former, on account of the limitations of 0 described at the top of this page.) The differentiation described above is not here shown, but was calculated in the problem. The result of the whole calculation has been, then, to produce the green curve in the figure (3a), which corresponds to the green 
line in the $W$ plane. The other curves can be drawn mechanically by simply permuting the green curve around the origin at intervals of $\frac{2 \pi}{5}$, as has already been shown.

To get correspondences of regions, and connections of sheets, we shall cut the $\mathbb{W}$ plane outward from the points marked $A, B, C, D$, and $E$, toward infinity. These points correspond to the points marked similarly in the $Z$ plane, and the cuts described are indicated by the dotted Iines in both planes. It is possible to circulate around any point marked 0 in elther plane without crossing a dotted Iine, but it is impossible to circulate intirely around any of the points marked $A, B, C, D, E$, without crossing a dotted line. The sheets in the $Z$ plane are therefore as indicated by the Arabic numerals, and each sheet includes the space between two dotted lines extending outward from the origin to infinity, and each sheet is seen to be so dra:in that any point can be reached from any other point in it without coossing a dotted line. To make identification more convenient, I have numbered the reglons I, II, III, IV, and $V$, and have said that region is identified by the clear orange line, region II by the clear purple line, etc.; it is then easy to identify the regions around any of the 0 points by noticing the $k$ ind of line in the region. Having thus identified regions and sheets, it can be seen that sheets 3,4 , and 5 extend across the dotted blue line unbroken, while sheets 1 and 2 connect with each other across this line; sheets 1, 4, and 5, connect across the dotted red line unbroken, while sheets 2 and 3 connect with each other across this line. In a similar manner connections across all the other lines can be determined, and 
are as indicated in the figure. (Fig. $3 b$ ).

It is interesting to note in the figures for this function that the areas in either plane are bounded by the same kind of lines- this is true in general, of course, but it is worth especial notice here to see the five areas in each case, in the $Z$ plane, which corfespond to the single area in the $W$ plane. In both the complicated analytic cases discussed, it is seen that the number of sheets is equal to the order of the function. This is true in general, as can be shown by theory of Riemann Surfaces in general, which need not be discussed here. 


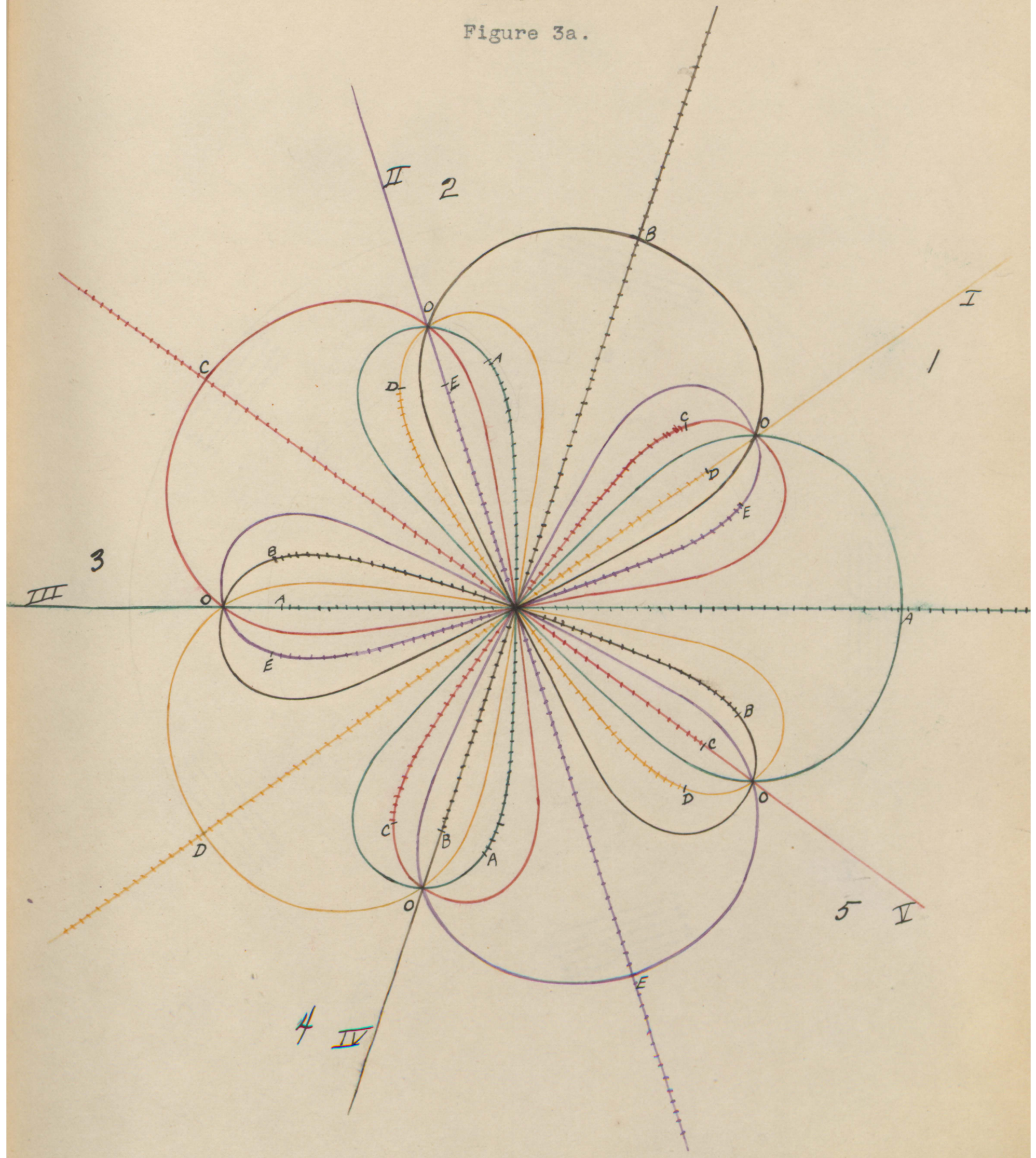


Figure $3 b$.

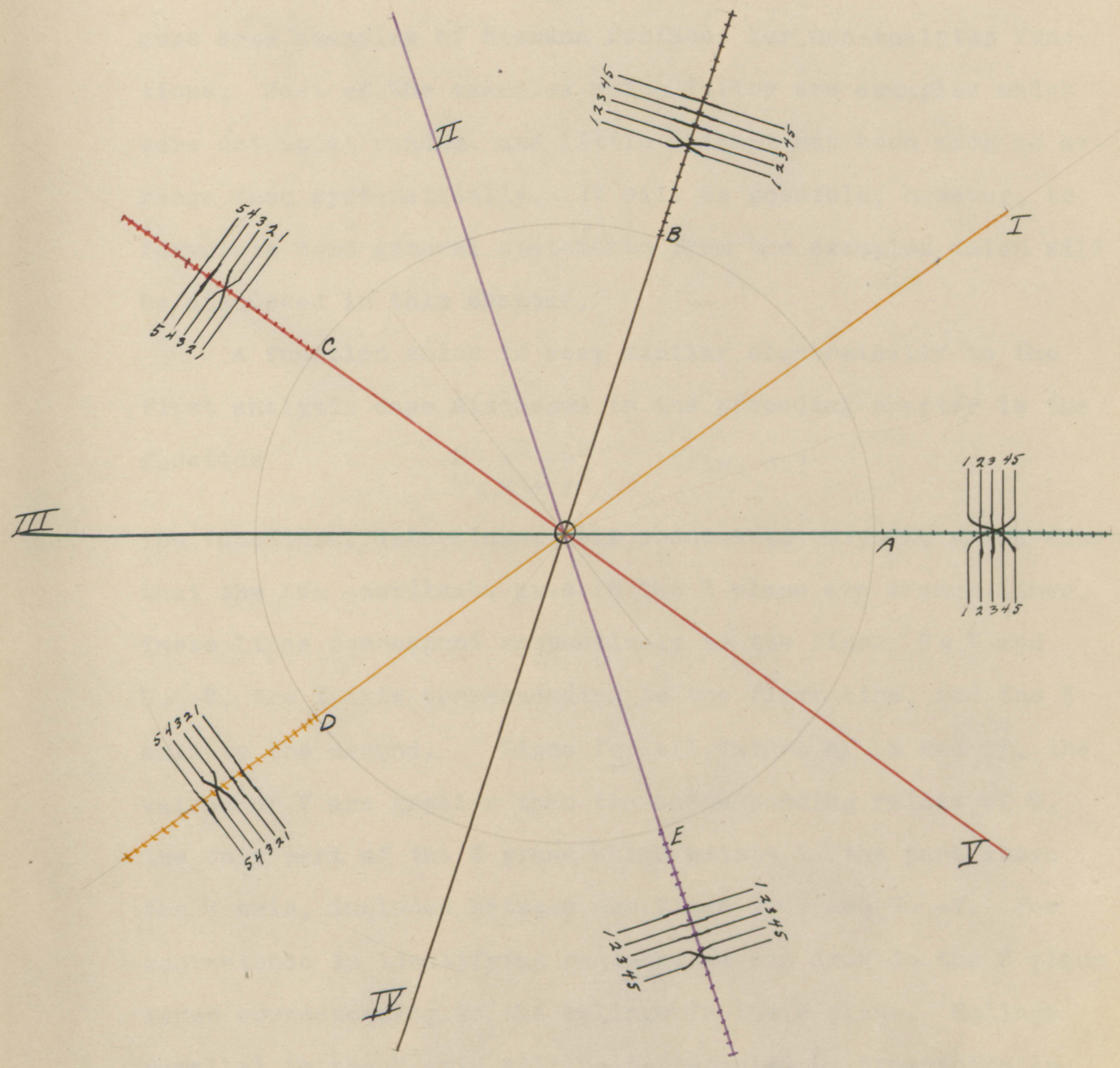




\section{CHAPTER II}

In the preceding chapter we discussed some examples of Riemann Surfaces for analytic functions. Te shall now discuss some examples of Riemann Surfaces for non-analytic functions. Most of the examples which follow are examples which were set up at random, and little attempt has been made to arrange them systematically. It will be possible, however, to formulate some general statements from the examples which will bo discussed in this chapter.

A function which is very similar algebraically to the first analytic case discussed in the preceding chapter is the function

$$
\begin{aligned}
& \mathrm{J}=\mathrm{x}^{2}-\mathrm{y}^{2} \\
& \mathrm{~V}=\mathrm{x}^{2}+\mathrm{y}^{2}
\end{aligned}
$$

The functional determinant here reduces to $8 x y=0$, which shows that the two coordinate axes in the $z$ plane are branch lines. These lines correspond respectively to the lines $U=V$ and $U=-V$, the $X$ axis corresponding to the first line, and the $Y$ axis to the second. Since for all values of $x$ and $y$, the values of $V$ are greater than the corresponding values of $U$, the only part of the $\mathbb{W}$ plane which exists is the part above the $U$ axis, included between the lines $U=V$ and $U=-V$. For convenience in identifying regions, we can draw in the $z$ plane lines corresponding to the rulings in tila $\mathrm{W}$ plane. Rulings parallel to the $V$ axis will be represented by hyperbolas in the $Z$ plane, positive values of $U$ being hyperbolas axial to the $X$ axis, and negative values of $U$ being hyperbolas axial to the $Y$ axis. The lines parallel to the $U$ axis, and above 1t ( the lower part of the $\mathbb{W}$ plane being non-existent) 
appear as circles in the $Z$ plane, with the origin as center. These corresponding lines are drawn in similar styles of lines, and are easily identifled. To determine the manner of connection of sheets, we shall trace the path of a point in the $\pi$ plane as the corresponding point makes a complete circuit of the $Z$ plane. Starting at the intersection of one of the red circles and the line $y=x$, (this line corresponding to the line $U=0$ ) and moving along the circle in a clockwise direction, we reach the dotted green line. In the $\mathbb{W}$ plane, this is the limit of motion in this direction, and we are forced to retrace the path, moving along the same horizontal line in the $\mathbb{W}$ plane, but in the opposite direction, as we move along the same circle in the same direction in the $Z$ plane. Considering that we started in the first sheet, we are now in the fourth sheet, using the accompanying diagram and numbering. Continuing along the same circle in the same direction, we reach the crossed green line, which is again the limit of motion in this direction, and we are forced into another sheet, which is the third, to correspond to the numbering of sheets in the diagram. Further motion along this circle, then, means retracing the path along the red line in the 7 plane, moving now from teft to right along the horizontal line. Continuing the motion in the $Z$ plane, we reach again the dotted green line, and we are forced into another sheet of the $\overline{7}$ plane, this time the second; and finally, we shall return to the first plane after crossing the crossed green line. It is seen that each sheet of the $W$ plane 


$$
{ }_{y}^{\text {Figure }} 4 .
$$

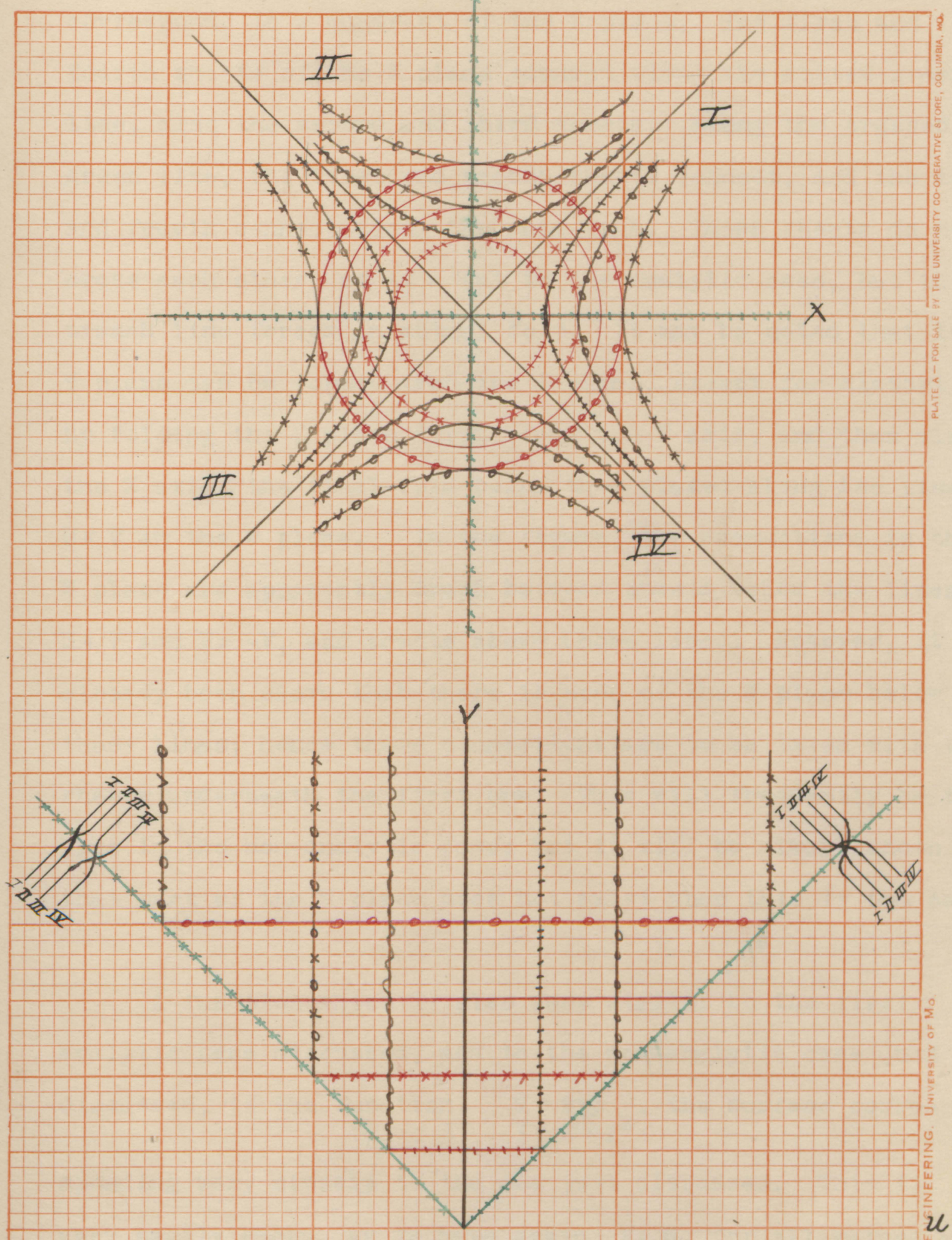


corresponds to a quadrant of the $Z$ plane. The first and fourth sheets of the $W$ plane connect along the dotted green line, also the second and third sheets. Along the crossed green line, the first and second sheets connect, and the third and fourth connect along the same line. These sheets do not cross each other, as at a cut in the analytic case, but the branch line forces an actual fold. It may be noted that the 7 plane can be formed from the $Z$ plane, except for distortion, by folding the $Z$ plane first along the $Y$ axis, and then along the $\mathrm{X}$ axis, the final fold being tinen placed along the line $U=V$ in the plane. In the figure, the connections in the W plane are apparently drawn between sheets below the lines $U=V$ and $U=-V$, and sheets above those lines. However, it has

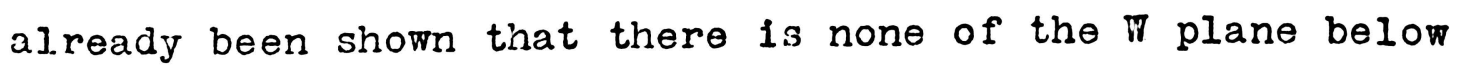
these lines; hence these connections are to be taken with the sheets underneath the first one. This will frequently be the case in the non-analytic case; but it will be always shown that part of the $\$$ plane does not exist when this is the case.

A function which is also very similar to the first analytic case discussed, and to the non-analytic case just

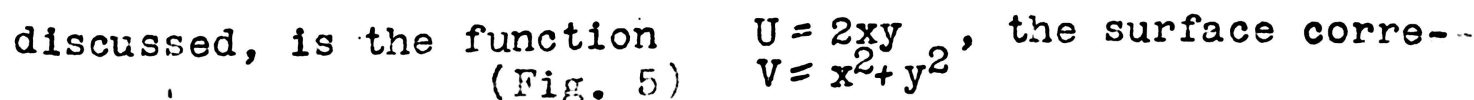
sponding very closely to the one just discussed. This is very easily shown, as we have again $V=U$, for all values of $x$ and $y$; it is also seen that the tmo surfaces must be very much alike, for the value of $O$ here is precisely the same 
Figure 5 .

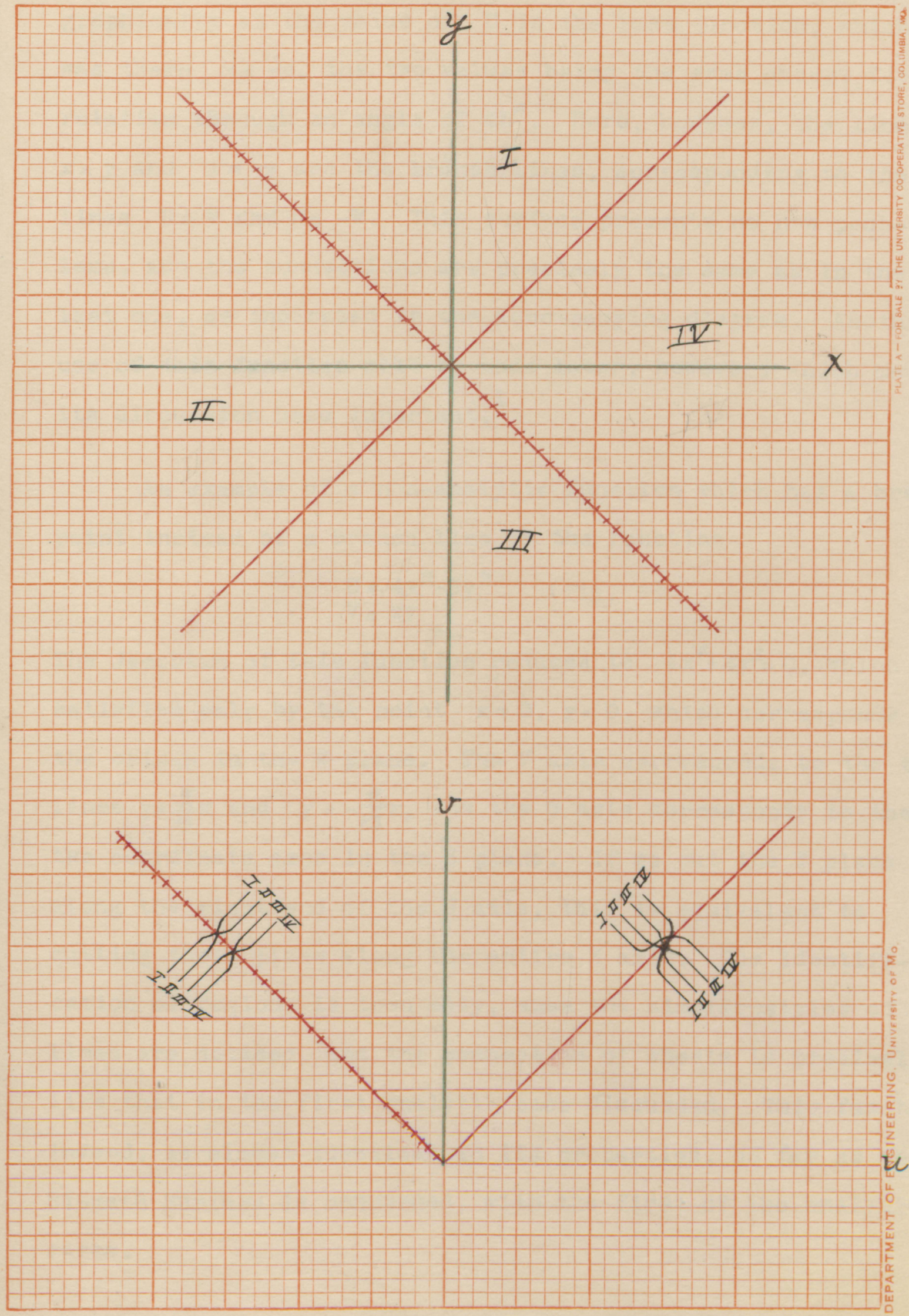


graphically as the value of $U$ in the last function, which was there $x=-y$, the two values being the same except for rotation through an angle of $45^{\circ}$. Here, however, the lines $x=y$ and $x=-y$ are the branch lines, corresponding to the lines $U=V$ and $U=-F$. This surface presents no new problems, and the connections are procisely the same as in the last one, as indicated in the accompanying diagram. Anotiner use of this function will be made later, however, which makes its discussion here important. ${ }^{1}$

An example of a non-analytic function which has a branch point instead of a branch line is the function

$$
\begin{aligned}
& U=4 x^{2}-3 y^{2} \quad(F i \xi \cdot 6) \\
& V=x y
\end{aligned}
$$

The Jacobian of this function reduces to $4 x^{2}+3 y^{2}=0$, which is satisfied only by the point $x=0, y=0$, which corresponds to the origin in the $\mathrm{V}$ plane. The $\mathrm{V}$ axis corresponds to the two lines $y=\frac{2}{\sqrt{3}} \mathrm{x}$ and $\mathrm{y}=-\frac{2}{\sqrt{3}} \mathrm{x}$, and the $\mathrm{U}$ axis corresponds to the two coordinate axes in the $Z$ plane. If we cut the 7 plane along the right end of the $U$ axis, this is equivalent to cutting the $Z$ plane along the entire $X$ axis. The identification of regions is then easy, when the similar styles of Ines are noticed, and the connection of sheets is precisely Iike that in the first analytic case discussed. This function, indeed, is almost identical in 1 ts properties, so fur as the Riemann Surface is concerned, with the first of the analytic cases. 
Figure 6.

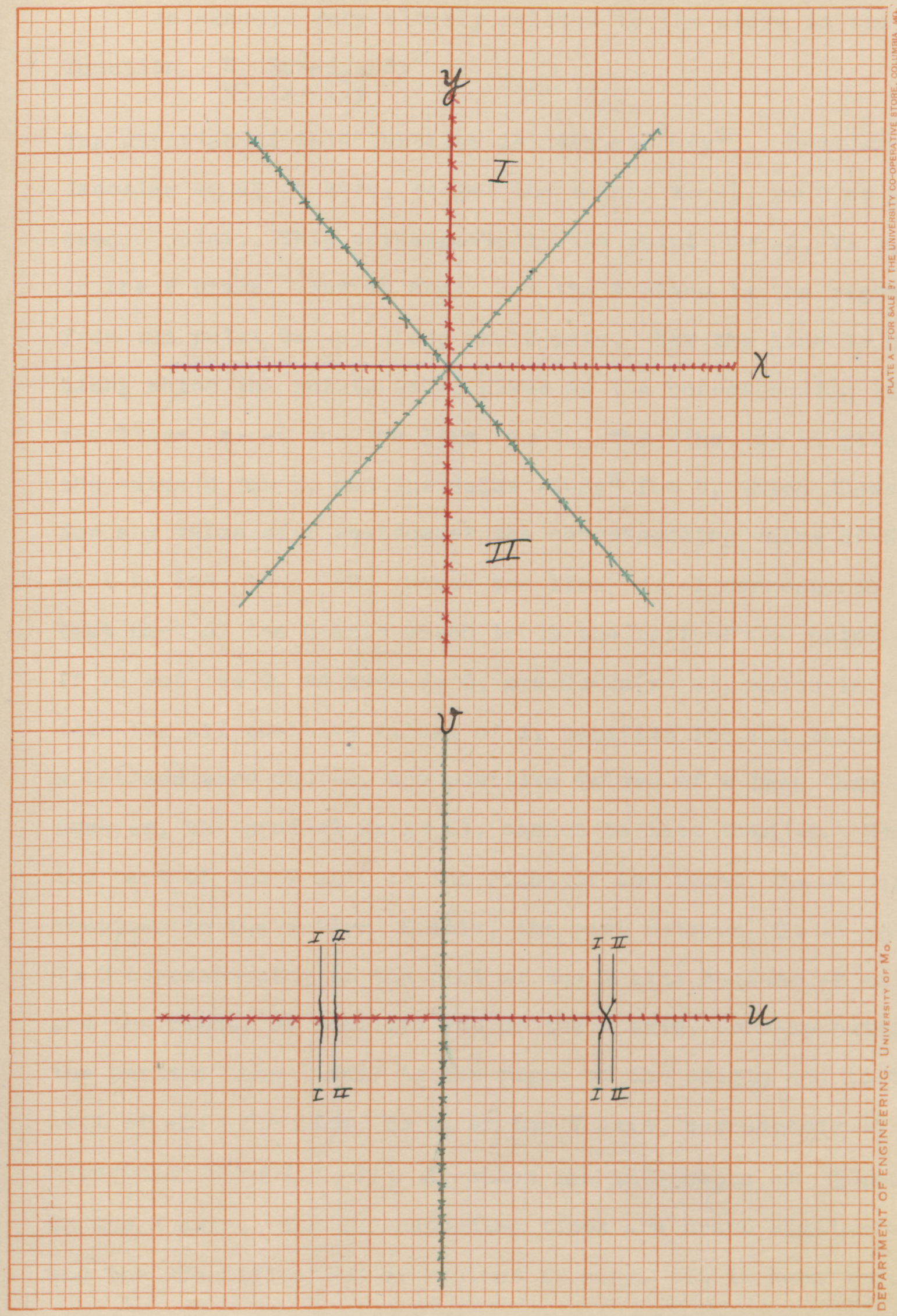


An example very closely related to the above is the function $\begin{array}{ll}U & 2 x^{2}+4 x-3 y^{2} \\ V & (x+1) y\end{array}$

The Jasobian of this function is $4(x+1)^{2}+6 y^{2}$. Setting this Jacobian equal to zero, we get the point $x=-1, y=0$ as the branch point. This corresponds to the point $U=-2, V=0$, in the $W$ plane. Now we shall cut the $W$ plane to the right from the point $U=-2$, along the $U$ axis. Now the Iine $V=0$, along which the cut is made, gives as the corresponding IInes, the lines $x=-1$ and the line $y=0$. The line $x=-1$ gives the value 0 for $V$, and the value $-2-3 y^{2}$ for $U$, which shows that for any value of $Y$ whatsoever the corresponding value of $U$ will be less than -2 . Hence the line $x=-1$ corresponds to that part of the $U$ axis which is to the left of the point $U=-2$. If we put $y=0$, we have $v=0$, and $\delta=2 x(x+2)$, wh1ch is positive for all positive values of $x$, negative for all values of $x$ between zero and -2 , and positive values of $x$ less than -2 . However, the product $2 x(x+2)$, cannot be less than -2 . Hence the part of the $U$ axis to the right of the point $U=-2$ corresponds to the entire $X$ axis, the part between $U=-2$ and $U=0$ corresponding to the part of the $X$ axis between -2 and 0 , and the right, of positive end of the $U$ axis, corresponding to the parts of the $X$ axis to the right of the origin and to the left of -2 . The line $U=0$, or the $V$ axis, corresponds to the hyperbola $2(x+1)^{2}-3 y^{2}=2$, which has intercepts of 0 and -2 on the $X$ axis. The corresponding lines are drawn similarly, and the identification of regions is easy. It is seen that 
Figure 7.

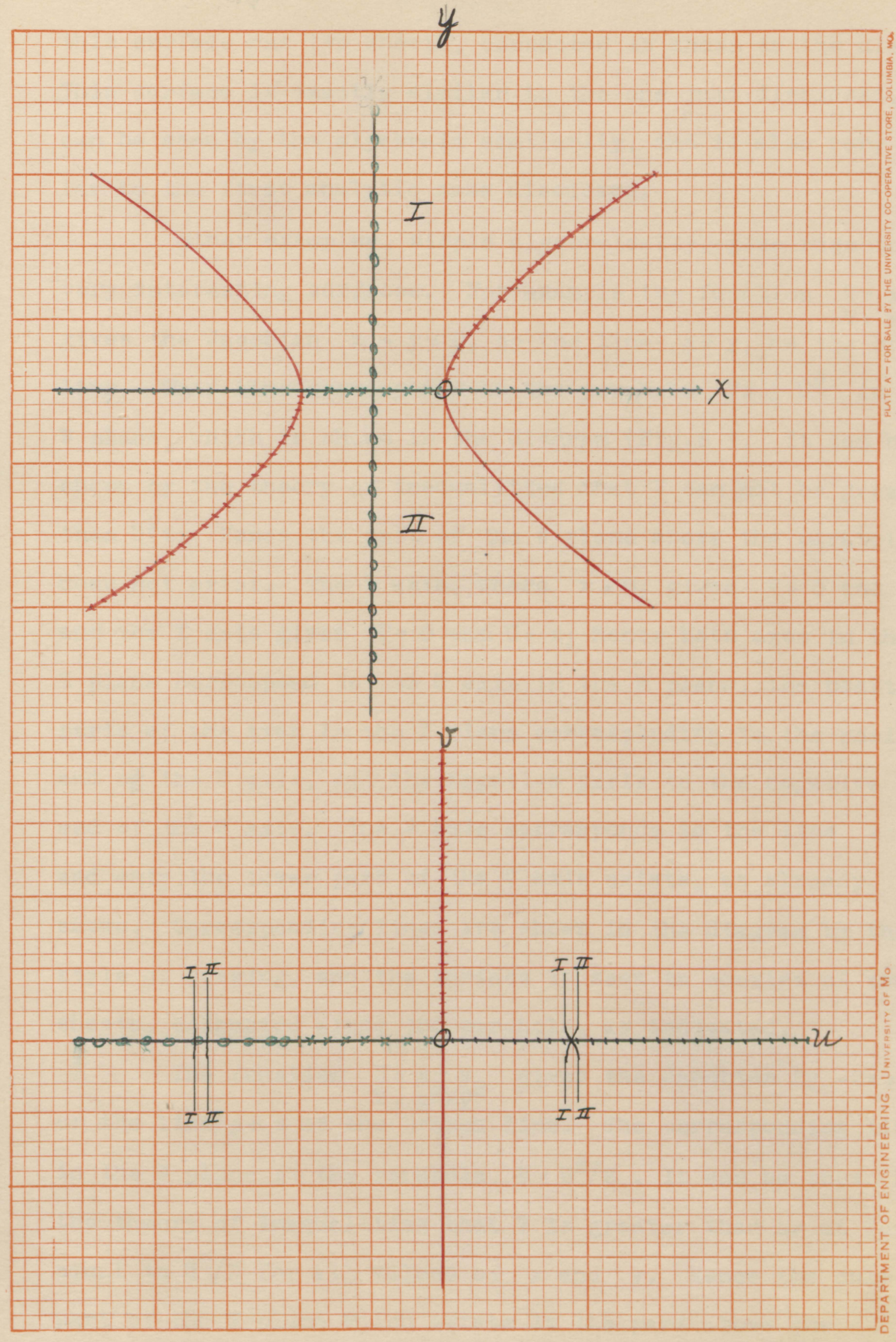


this surface has two sheets, connected as shown in the diagram. This surface is exactly similar to the one just discussed, as can be seen by the algebraic similarity, and is also very similar to the first analytic case discussed.

Other examples, simple algebraically, give rise to some very interesting surfaces. For example the function $U=x+y$ gives the Jacobian $x-y=0$, or the line $x=y$, as the branch line. This line corresponds to the parabola $V=\frac{1}{4} U^{2}$ in the Tiane, which is shown in the diagram (Fig.8). It can be shown by the theory of inequalities that $\mathrm{V}$ can not be greater than $\mathrm{U}^{4} 4$. Hence the part of the $\mathrm{W}$ plane above the parabola does not exist. The $Y$ axis, where $x=0$, gives $V=0$ and $U=y$, and the $X$ axis gives $V=O$ and $U=x$; hence both axes in the $Z$ plane correspond to the $U$ axis in the $W$ plane, the positive parts of the axes in the $Z$ plane corresponding to the positive end of the $U$ axis, and the negative parts to the negative end of the $U$ axis. The $V$ axis corresponds to the line $x=-y$, and only the lower part of the $\mathrm{V}$ axis exists, as already shown. Corresponding lines are shown in the diagram by the style of drawing, and the surface is seen to consist of two sheets, connected along the parabola, as indicated.

The function $\begin{aligned} & U=x+y \\ & V=x^{2}+y^{2}\end{aligned}$ gives the Jacobian $2 y-2 x=0$, or the line $\mathrm{y}=\mathrm{x}$ as the branch line. The corresponding line in the $\pi$ plane is the parabola $V=U^{2} / 2$. It can be shown by the theory of inequalities that $V$ is greater than or at least equal to $U^{2} / 2$ for all values of $x$ and $y$. Hence the only part of the $W$ plane which exists is the part above the 


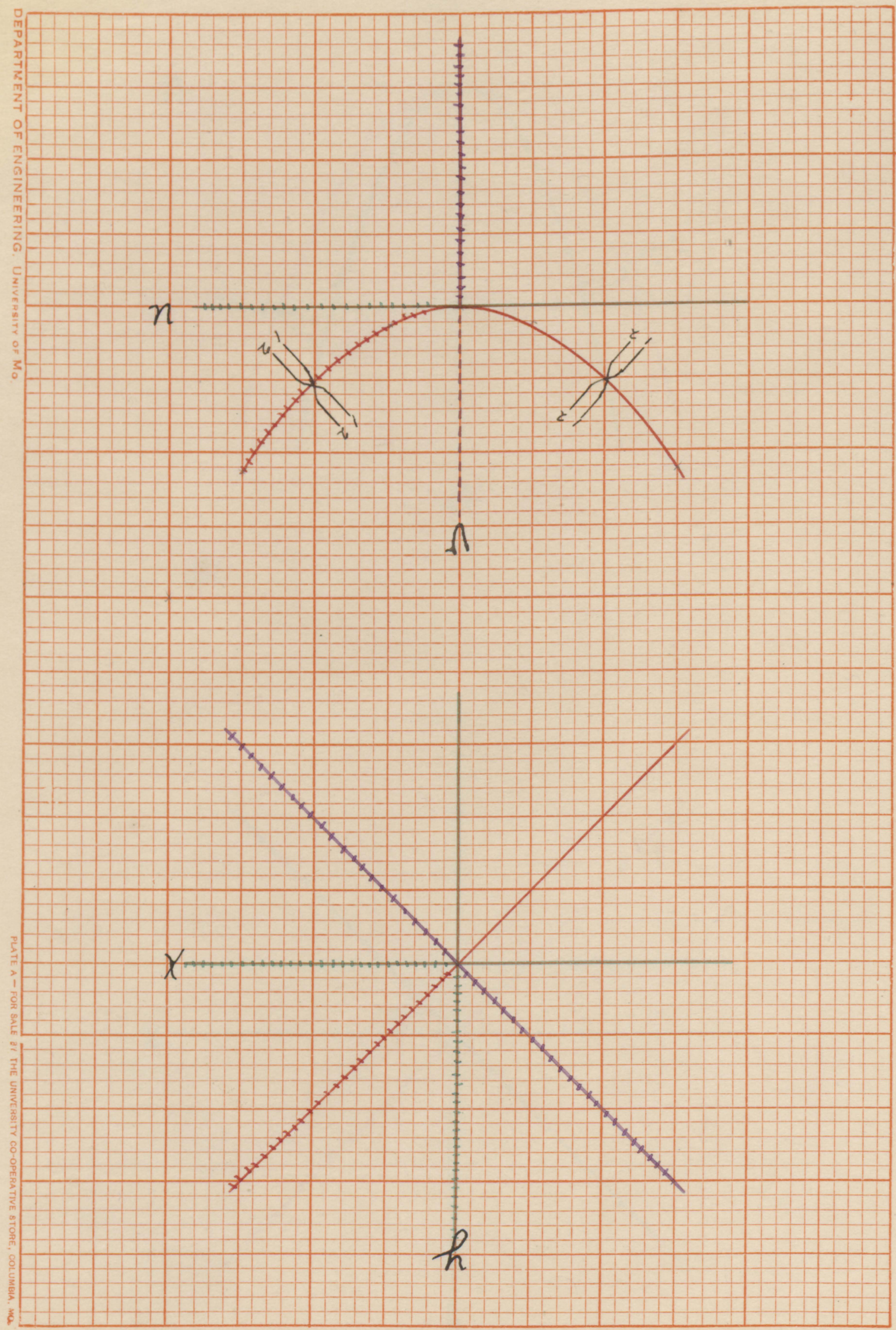

-8 องกริโน 

parabola described. If $x$ and $y$ are both positive, as for the part of the line $y=x$ which lies in the first quadrant, $U$ is positive; for values of $x$ and $y$ which lie in the third quadrant, $U$ is negative. Hence the portion of the line $y=x$ which lies in the first quadrant corresponds to the right branch of the parabola in the $W$ plane, and the part of the Iine $\mathrm{y}=\mathrm{x}$ which lies in the third quadrant corresponds to the left branch of the parabola. The axes in the $Z$ plane correspond to the parabola $V=U^{2}$ in the $W \cdot$ plane, the positive ends of the axes corresponding to the right branch, and the negative ends corresponding to the left branch. Tho line $U=0$, or the $V$ axis, corresponds to the line $y=-x$. It is then seen from the figure that the part of the $W$ plane above the parabola drawn in red- the other part of the $W$ plane being non-existent- consists of two sheets, connected along the red parabola in the manner indicated. (Fig. 9)

$$
\text { The function } \begin{aligned}
& U=x^{2}+y^{2} \\
& V=x^{4}+y^{4}
\end{aligned} \text { Gives the Jacobian } 8 x y\left(y^{2}-x^{2}\right)
$$

which shows that the branch lines are the axes and the lines $y=x$ and $y=-x$. The $z$ plane is therefore divided into elght sheets, or regions. The lines $y>x$ and $y=-x$ correspond to the parabola $V=U^{2} / 2$, and the axes correspond to the parabola $V=U^{2}$. By the theory of inequalities it can be shown that $V$ can not be less than $U^{2} / 2$ nor greater than $U^{2}$. Hence the only part of the $\mathbb{W}$ plane which exists is the part between the two parabolas, and in the first quadrant, since both $U$ and $V$ are positive for all values of $x$ and $y \cdot(F \mid \xi .10)$ 
Figure 10.

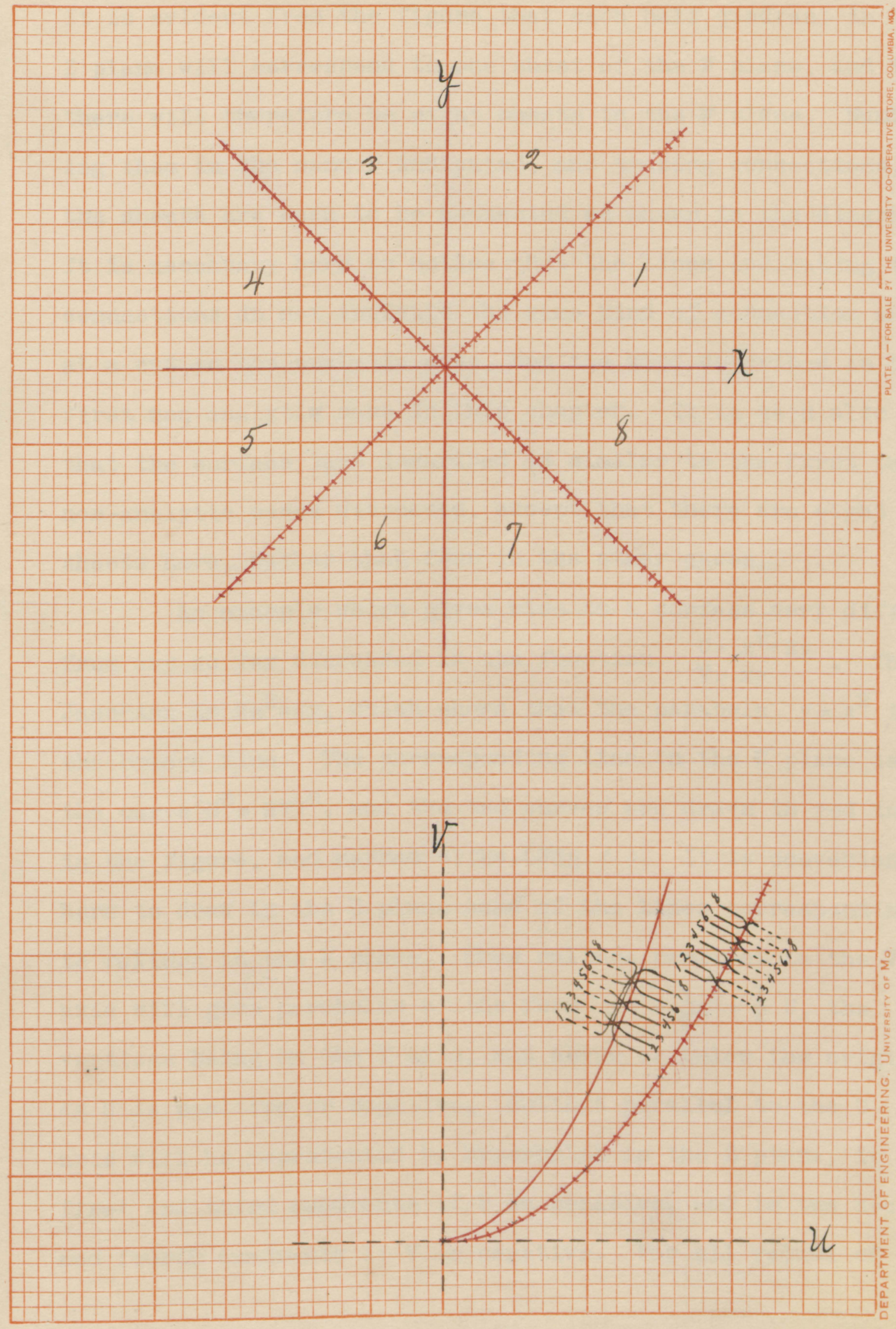


It is evident from the figure that each half quadrant in the $Z$ plane will correspond to one sheet of the $W$ plane, and that there will therefore be eight sheets in the $\mathbb{W}$ plane. Numbering them in the manner indicated, and tracing connections, the sheets are seen to be connected as shown in the diagram. It is necessary here to notice that the lines indicating the connections do not indicte the same kind of connection as at a cut in the analytic case, but the kind described in the first non-analytie case in this chapter. It is seen also that the surface just described is simllar to the flrst two non-analytic cases discussed, except that there are eight sheets here, rather than four in the other cases.

An example wich gives rise to a surface very different from any so far discussed is the function $\begin{aligned} & U=x^{2}+2 y \\ & V=x y\end{aligned}$ Jacobian is $2 x^{2}-2 y$, which shows that the parabola $y=x^{2}$ is the branch line. The corresponding line in the $\mathbb{W}$ plane is the curve $U^{3}=27 V^{2}$, and the two curves are drawn in red ink in the figure, and marked alike.(Fig.//). It is convenient here to draw in the $Z$ plane some of the lines corresponding to rulings in the $W$ plane. Thus mulings parallel to the $V$ axis will give parabolas of the form $y=-\frac{1}{2} x^{2}+\frac{1}{2} U$, the vertices being on the $Y$ axis, and above or below the origin as $U$ is positive or negative. Rulings parallel to the $U$ axis will be represented by hyperbolas in the $Z$ plane, in the first and third quadrants if $\mathrm{V}$ is positive, and in the second and fourth quadrants if $\mathrm{V}$ is negative. For all values of 
U greater than $3 \mathrm{~V}^{\frac{2}{3}}$, the corresponding curves in the $Z$ plane are seen from the diagram to intersect three times, while for values of $U$ less than $3 V^{\frac{3}{3}}$ the corresponding curves intersect but once. For values of $U$ equal to $3 V^{\frac{2}{3}}$, the corresponding curves are tangent along the red parabola. Since $U$ is expressed by an even power of $V$, the above remarks apply to negative as well as positive values of $v$. Now all values of U greater than $3 V^{3 / 3}$ are found to the right of the red curve in the $\pi$ plane, in the wedge shaped region bounded by tho two branches of this curve, and all values of $U$ less than $3 V^{2} \frac{3}{3}$ are to the left of the red curve. The wedge shaped region is therefore composed of three sheets, while the remainder of the $\mathbb{W}$ plane consists of only one sheet. The branch line represents a fold here, just as in the preceding cases, and we can pass from the fourth quadrant of the $W$ plane to the first quadrant only by passing alternately to second and third sheets. The connections are rather difficult to indicate by a diagram, and a paper model of this surface is inserted. It is seen to differ very sharply from any surface so far discussed. (Plate I. Fig.11.)

We may study analytic transformations of some of the non-analytic functions discussed. As an example, the function $W=U+V 1=2 x y+\left(x^{2}+y^{2}\right) 1$, the second example in this chapter, gives, when squared, $-\left(x^{2}-y^{2}\right)^{2}+4 x y\left(x^{2}+y^{2}\right) 1$. Calling the real and imaginary parts $R$ and $S i$ respectively, and setting 


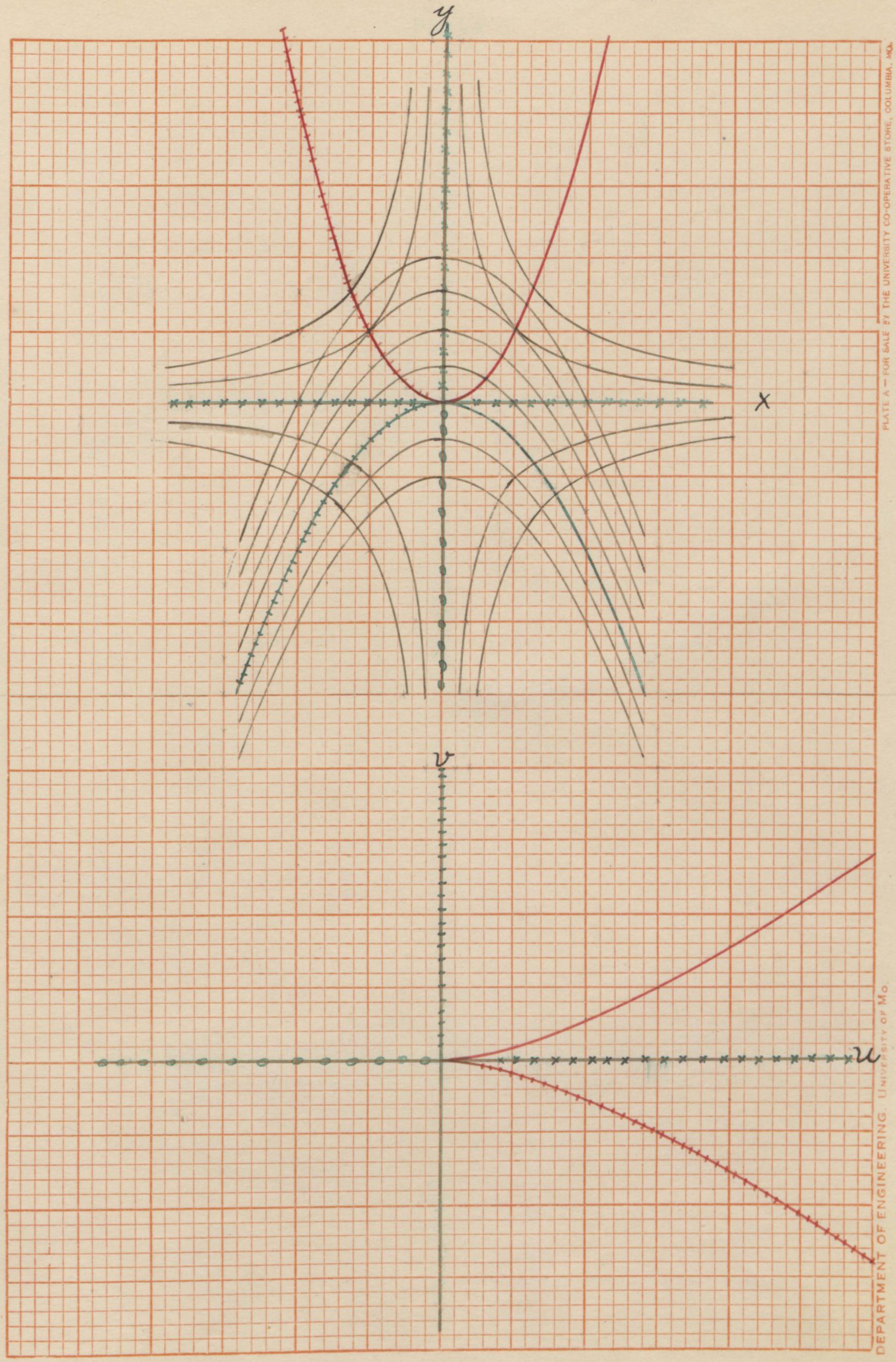


Plate I

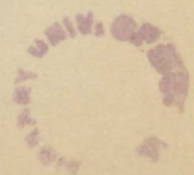


up the Jacobian $\frac{D(R, S)}{D(x, y)}$, we have $\left(x^{2}-y^{2}\right)\left(x^{4}+6 x^{2} y^{2}+y^{4}\right)=0$, which is satisfied by the Iines $y=x, y=-x$, and the point $x=0, y=0$, which shows that the function has branch lines and a branch point. The branch point is on both the branch Iines, and therefore this point must have properties different from the properties of an ordinary point on a branch line. This distinction between an ordinary point on a branch Iine, and a branch point, will be commented on shortly. The original function shows that $R$ can not be positive in value, and the left half only of the RS plane can exist. $S$ will be negative if $R$ and $S$ be unlike in sign, and positive if $R$ and $S$ be alike in sign; hence the first and third quadrants of the $Z$ plane will be transferred to the upper half of the RS plane, and the second and fourth quadrants to the lower half. Furthermore, $R$ and $S$ are expressed as symmetric functions of $x$ and $y$; therefore any two points which are situated with respect to the lines bisecting the quadrants in the $Z$ plane will go to the same point in the RS plane. Thus there are elght sheets in the RS plane, four on the upper half, and four on the lower half. Both axes in the $Z$ plane go into the $R$ axis, the Iine: $y=x$ to the upper end of the $S$ axis, and $y=-x$ to the 10 wer half of the $S$ axis. The connections are then easy to trace, and are shown in the diagram. It is seen that the branch point prevents circulation around it, even in one plane, which is not true in general of a point on a branch line. The axes in the $Z$ plane are used as cuts in the above case, and are similar to the cuts in the analytic case. (Fig. 12) 
Figure 12.

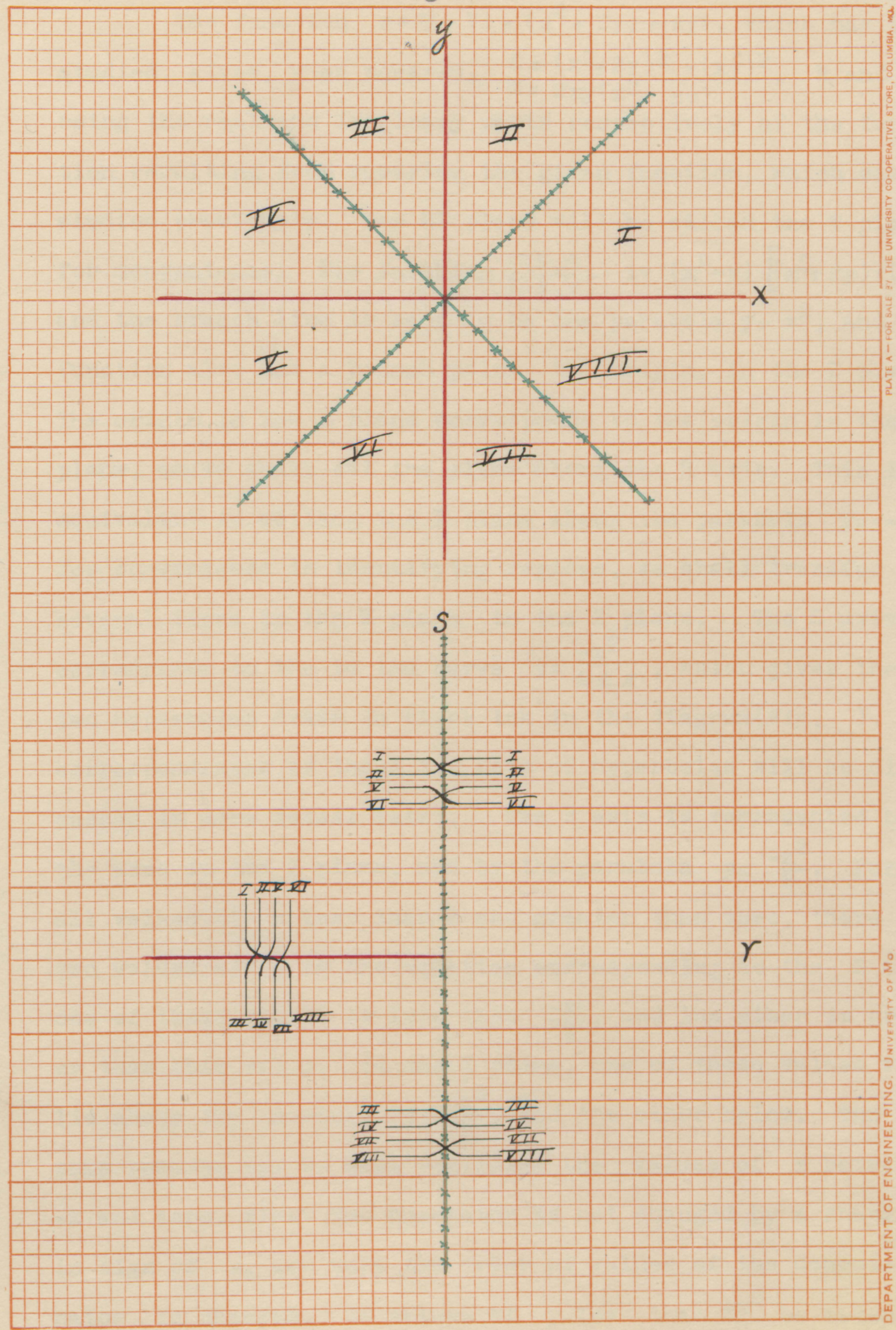


The function $w=U+V i=\left(x^{2}+2 y\right)+x y i$, which has been studied, gives, when squared, $\begin{aligned} & R=\left(x^{2}+2 y\right)^{2}-(x y)^{2}, R \text { and } S \\ & S=2 x y\left(x^{2}+2 y\right)\end{aligned}$ beling the real and imaginary parts respectively of the function resulting from squaring the given function, which was studied previously. The RS plane will be called tine $T$ plane for convenience. The Jacobian $\frac{D(R, S)}{D(x, y)}$ is $\left(x^{2}-y\right)\left[\left(x^{2}+2 y\right)+(x y)^{2}\right]$ and the branch line is seen to be the parabola $y=x^{2}$, and the function also has a branch point- the origin, in the $z$ plane. The origin in the $z$ plane corresponds to the origin in the $T$ plane, andwe shall cut the plane along the $R$ axis. This is equivalent to cuttine the $z$ plane along the parabola $y=-\frac{x^{2}}{2}$. For convenience in ldentifyling regions, we shall transfer several lines in the $\mathbb{Z}$ plane to the $Z$ plane. The $S$ axis corresponds to the to the curve $y=\frac{-x^{2}}{2 \pm x}$, which gives the two hyperbolas drawn in black ink in the diagram(Fig.). The correspondences of these two sets of hyperbolas will be discussed in more detall later. The $R$ axis is represented by the axes in the $\mathrm{z}$ plane, and by the parabola $y=\frac{-x^{2}}{2}$. For all values of $x$ less than 2 and greater than zero, there are two values of $y$ in the equation corresponding to the $S$ axis; one of these values is less than $\frac{-x^{2}}{2}$, and the other is greater than this value. In the $Z$ plane, one of these values will lie above the parabola $y=\frac{-x^{2}}{2}$, and the otiner below. The same remarks may be made regarding the values of $x$ between -2 and zero, since the numerator is not changed by a change in sign of $x$, and only the order of values of the denominator is changed. If values of $y$ less than $\frac{\dot{4} x^{2}}{2}$ be taken 
with positive values of $x$, the value of $S$ will be positive, and if values of $y$ greater than $\frac{-x^{2}}{2}$ be taken with negative values of $x$, the values of $S$ will be positive also. A similar argument will establish correspondences for negative values of $\mathrm{S}$. For values of $\mathrm{x}$ greater than 2 or less than -2 , the values of $y$ will lie either entirely above the $\mathrm{X}$ axis, or above the parabola $\mathrm{y}=\frac{-x^{2}}{2}$, and below the $\mathrm{X}$ axis. It is then easy to determine the correspondences of the hyperbola mentioned above, and the style of marking shows the correspondence. The parabola $y=\frac{-x^{2}}{2}$ also corresponds to the left end of the $R$ axis, as can be shown by substituting this value in the original expression for $R$. The axes in the $Z$ plane are shown by substitution to correspond to the right end of the $R$ axis.

The parabola $y=x^{2}$ is found by substitution to correspond to the equations $R=x^{4}\left(9-x^{2}\right)$, and $S=6 x^{5}$. Solvinf these equations by eliminating $x$, we have the relation $R=\left(\frac{5}{6}\right)^{\frac{4}{3}}[9$-(新] - The nature of this curve may be in some measure determined by determining the maxima or minima, and the intercepts on the axes. The derivative $\frac{d R}{d S}$, set equal to zero, locates the maximum points with respect to the $R$ value; these are found at $R=108$ and $S= \pm 216 \sqrt{6}$. The values of $S$ for $R=0$ are 0 and \pm 1458 . The function, plotted on a very small scale, is therefore represented by the red curve in the $T$ plane, and this corresponds to the parabola $y=x^{2}$. In the equations in which $R$ and $S$ are expressed in terms of 
the parameter $\mathrm{x}$, the following relations can be shown:

$$
\begin{aligned}
& \text { For } 0<x<3, R \text { and } S \text { positive. } \\
& \mathrm{x}=3, \mathrm{R}=0, \mathrm{~S}=1458 \\
& x>3 . R<0, S>1458 \\
& \text { For } 0>x>-3, R>0, S<0 \\
& x=-3, R=0, S=-1458 \\
& \mathrm{x}<-3, \mathrm{R}<0, \mathrm{~S}<-1458 \text {. }
\end{aligned}
$$

Therefore the part of the red parabola in the $Z$ plane to the right of the point $x=3$ and to the left of the point $x=-3$ correspond to the parts of the red curve in the $T$ plane to the left of the $S$ axis. Furthermore, the two hyperbolas which intersect the red parabola in the $Z$ plane at the points $x=3$ and $x=-3$ can represent only the parts of the $S$ axis above the value $S=1458$ and below $S=-1458$ respectively, for it is impossible to start from either of these points of intersection and reach the origin by traveling along a black line; the only regions which can be reached along the black line are between 1458 and infinity in the first case and between -1458 and minus infinity in the other case. If we now trace the path of a point around the origin in the $z$ plane, and trace the corresponding path in the $T$ plane, we shall find that the red line is a fold of the same sort as was the red curve in the original function, of which this is the second power. The connections are not easy to indicate by a diagram, and a paper model is inserted. (Fie. 13.) (Ilate II.) 
Figure 13a.

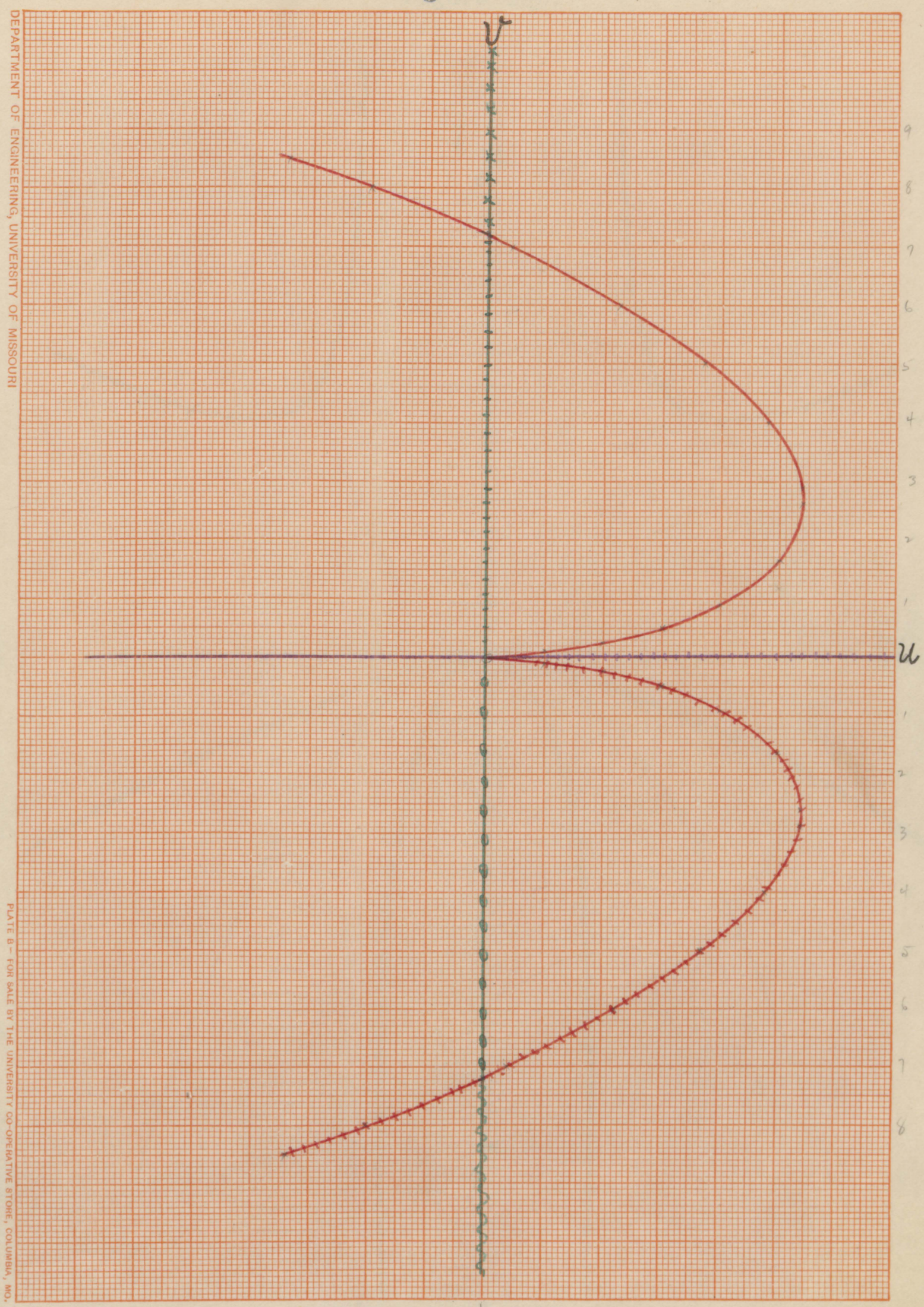


Figure 13b.

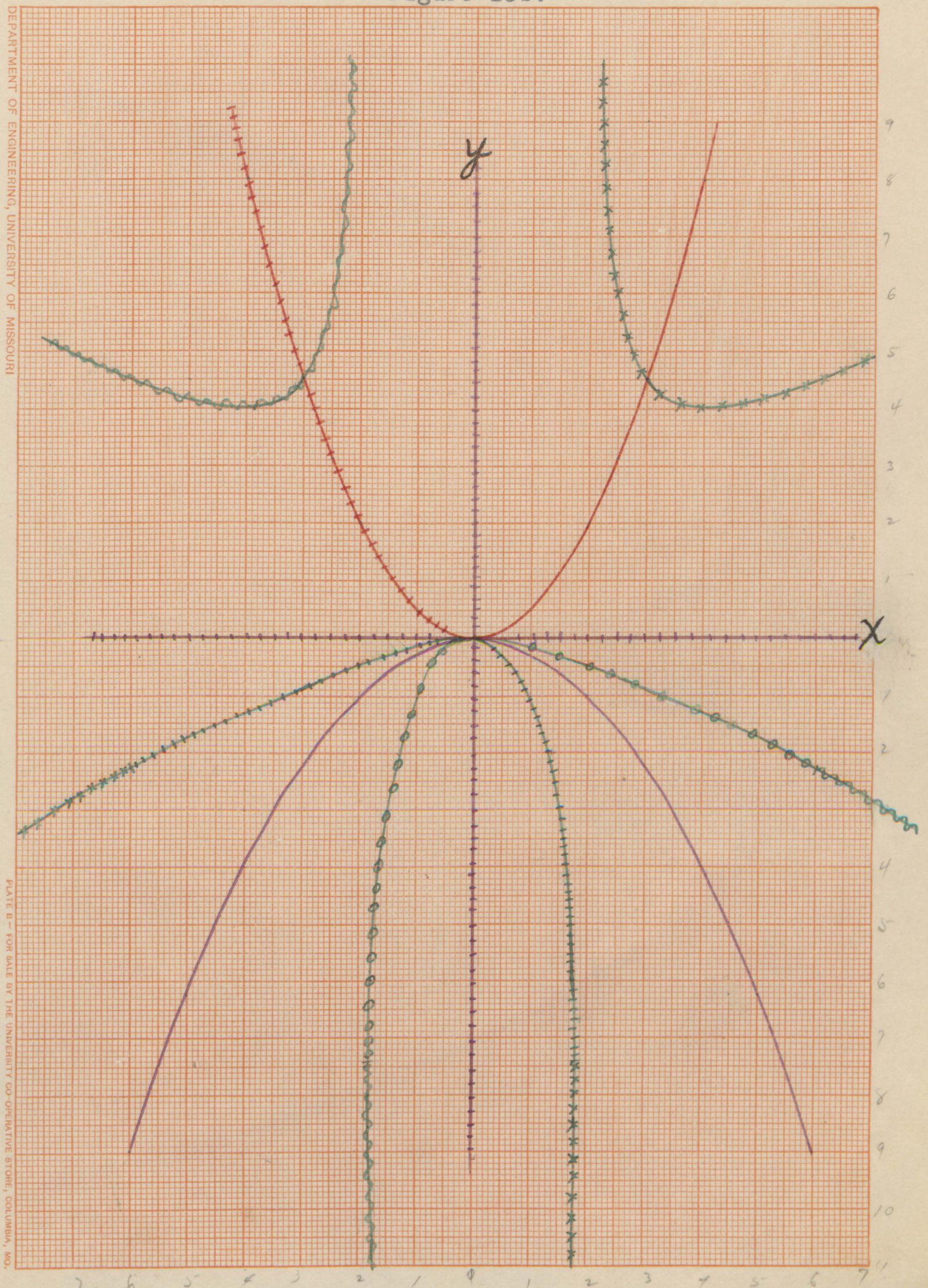


CHA PTER III.

In the preceding chapter, examples were set up and their surfaces discussed in considerable detall with no attempt to make their treatment systematic, and with no attempt to follow a fixed plan of setting up the functions. In this chapter at least two methods of setting up functions will be discussed-that is, methods of setting up functions which shall show branch lines or points previously determined. The branch line, and the consequent fold in the surface, suggeste the idea of edge of regression. An edge of regression is defined as the envelope of a certain family of curves which do not cross the envelope, as a cuble, for instance, might do. By means of certain conventions which shall be adopted, the 1dea of sheets can easily be determined definitely.

If we consider that a curve of any desired form be so moved as to be always tangent to a certain other curve, the second curve becomes an envelope for the flrst, and in case the first curve does not cross the envelope, we have an edge of regression. At least two sheets may be sald to be generated on one slde of the edge of regression by the moving curve- as a parabolia moving always tangent to a straight line, for example, will generate two sheets on one side of the line, since each point in the plane on that side of the line will be reached twice by the parabola. If the moving curve be a quartic, for example, there will be four sheets, perhaps, 
generated on part of the plane, and only two on the rest, or it may generate only two sheets on any part of the plane. A few special cases will be discussed here.

Let us consider that a curve of the form $v f(u)$ be moved so that it will be always tangent to another curee of the form $v=F(u)$. Then $v=F(u)$ is the edge of regression for the curves of the form $v=f(u)$. If we define positive motion along $F(u)$, and positive motion along $f(u)$, we can loctte any point in the plane in the following manner: starting from any fixed point $P$ on $F(u)$, move along $F(u)$ until the point $\left(u_{0}, v_{0}\right)$, which is the point of tangency of $F(u)$ and one of the curves of the form $f(u)$, is reached. Now move along $f(u)$ from $\left(u_{0}, v_{0}\right)$ to the given point $(u, v)$ which was the point to be reached from $P$. Denote by $X$ the first motion described, and by $Y$ the second motion described. Then the coordinates of any point in the plane can be determined in terms of $X$ and $Y$, since $X$ is the arc length along $F(u)$ from $P$ to $\left(u_{0}, v_{0}\right)$ and $Y$ is the arc length along $f(u)$ from $\left(u_{\theta}, v_{0}\right)$ to $(u, v)$. Now we shall define positive motion along $F(u)$ as that motion which will place the tangent family on the right hand side, and positive motion along $f(u)$ as the motion in the direction which will not produce a cusp with positive 'motion along

$A(u)$. In general, or it may be said in all cases, the point $u_{0}$ is a function of $u$ and $v$, and the particular problem will

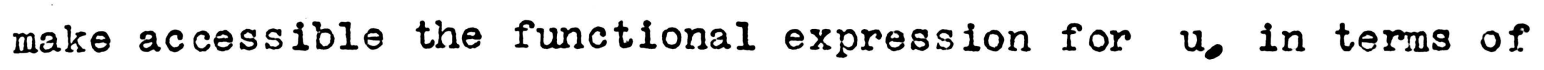
$u$ and $v$. As has been said, there will be more than one sheet 
generated, and it is altogether possible that there will be more sheets on some parts of the plane than on others.

Haring established these conventions, we shall proceed to set up a theory by which a function may be secured which will show any desired edge of regression. Let it be assumed that the edge of regression $v=F(u)$ is to be defined by tangent curves of the form $v=f(u)$. In general $f(u)$ will be a function of $u_{0}$ and $u$; hence we shall express $f(u)$ as $\theta\left(u_{0}, u\right)$, and this notation will be used for the tangent family. Now the function $x_{2}$ described above, will be represented by the integral $X=\int_{P}^{d} \sqrt{1+\left[F^{\prime}(m)\right]^{2}} d u$, and $Y$ will be represented by the integral $Y=-\int_{\mu}^{u_{0}} \sqrt{1+[\theta \text { ino, } \mu)]^{2}} d u$ It can be shown that the two functions $X$ and $Y$, defined by the two integrals, must together define a function $X+Y 1$ the edge of regression of which will be $v=F(u)$.; for,

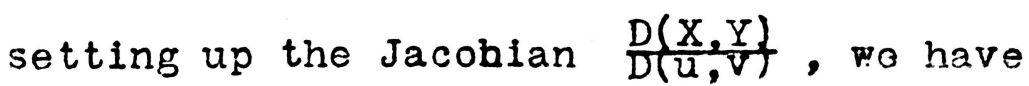
$\frac{\partial x}{\partial u}=\frac{\partial u_{0}}{\partial u} \sqrt{1+\left[f^{\prime}\left(u_{0}\right)\right]^{2}}+-\frac{\partial y}{\partial u}=-\int_{u}^{\mu_{0}} \frac{\theta^{\prime}\left(u_{0}, u\right) \frac{\partial u_{0}}{\partial u}}{\sqrt{1+\left[\theta^{\prime}\left(u_{0} u\right)\right]^{2}}} d u-\frac{\partial u_{0}}{\partial u} \sqrt{1+\left[\theta^{\prime}\left(u_{0} u_{0}\right)\right]^{2}}$
$\frac{\partial x}{\partial v}=\frac{\partial u_{0}}{\partial v} \sqrt{1+\left[f^{\prime}\left(u_{0}\right)\right]^{2}}$

$$
\begin{aligned}
& \frac{\partial y}{\partial v}=-\int_{u}^{u_{0}} \frac{\theta^{\prime}\left(u_{0} u\right) \frac{\partial u_{0}}{\partial v}}{1+\left[\theta^{\prime}\left(u_{0} u\right)^{2}\right.} d u-\frac{\partial u_{0}}{\partial v} \sqrt{1+\left[\theta^{\prime}\left(u_{0} u\right)\right]^{2}} \\
& \mathcal{J} \frac{(x, y)}{(u, v)}=-\frac{j u_{0}}{\partial v} \sqrt{1+\left[f^{\prime}\left(u_{0}, u\right)\right]^{2}} \sqrt{1+\left[\theta^{\prime}\left(u_{0}, u\right)\right]^{2}} \quad \therefore J\left(\frac{u_{1} v}{x, y}\right)=\frac{-1}{\frac{\partial u_{0}}{\partial v} \sqrt{1+f^{\prime 2}} \sqrt{1+\theta^{\prime 2}}} \\
& \text { How if } v=\theta\left(u_{0 .}, u\right) \\
& \frac{\partial \theta}{\partial u_{0}}=0 \quad \frac{\partial \theta}{\partial u_{0}} \frac{\partial u_{0}}{\partial v}=1 \quad \therefore \quad \frac{\partial u_{0}}{\partial r}=\frac{1}{\partial \theta} \quad \therefore J\left(\frac{u_{1}, v}{x, y}\right)=\frac{-\frac{\partial \theta}{\partial u_{0}}}{\sqrt{1+f^{\prime 2}} \sqrt{1+\theta^{12}}} \\
& \text { But } \frac{\partial \theta}{\partial x_{0}}=0 \text { is the cunditini for invelope. Therefre the mumersto } \\
& \text { sunt have a factor } v=F(x) \text {. }
\end{aligned}
$$


The abdve method of finding the edge of regression is the same as that used in the preceding chapter. In tiose examples, we have $U$ and $V$ expressed in terms of $x$ and $y$, and we set up the Jacobian $\frac{D(U, V)}{D\left(\frac{x}{y}\right)}$, which, equated to zero, gives curves in the $Z$ plane; the corresponding curves in the $\mathbb{W}$ plane are the branch lines, or edges of regression. If the equations be solved for $X$ and $Y$ in terms of $u$ and $v$, and the Jacobian $\frac{D(X, Y)}{D(u, V)}$ be set up, the resulting expression is the reciprocal of the first expression derived by the Jacobian, but expressed in terms of $u$ and $v .^{l}$ Hence if we set the reciprocal of this last Jacobian equal to zero, we have the edge of regression directly. In general, however, it is difficult or impossible to solve for $X$ and $Y$ in terms of $u$ and $v$, and the method of the preceding chapter is preferable. But in the theory of edgee of regression just worked out, we have the equations already solved for $X$ and $Y$ in terms of $u$ and $v$, and it would be impossible, in general, to express them in the form of the preceding chapter. The theory of the present chapter is preferable, and is easily applied, in this case.

Some examples which will be discussed illustrating the above theory are (a) a function which will have an edge of regression of the form $v=m^{2}$, defined by straight line tangents; (b) a function which will have an edge of regression of the form $v=m u^{2}$, defined by tangents of the form $v=-a u^{2}+b u+c$, where $a$ will be held constant, and $b$ and $c$ be allowed to vary; (c) a function which has an edge of regression a curcle of radius $r$, center at origin, defined by straight 
line tangents; and (d) a function which has as the edge of regression the circle $u^{2}=v^{2}+r^{2}$, defined by tangent circles of radius $R$, outside the circle of radius $r$. The last two cases are periodic, the case (c) belng singly periodic, with period $2 \pi r$, and case (d) doubly periodic, the periods being $2 \pi \mathrm{r}$ and $2 \pi \mathrm{R}$. In connection with the first example mentioned, and with the last two, it may be remarked that while the theory is perfectly general, yet it is possible at times to express $X$ and $Y$ by purely geometric means, without concerning one's self with the integrals defining $X$ and $Y$. Of course this equivalent to to the general method, since the integrals may be considered evaluated when we get the geometric expression for them.

The first example mentioned- the function which will have an edge of regression of the form $v=m u^{2}$, defined by straight line tangents, may be set up as follows. If any point, as $(u, v)$, outside the parabola, be taken, two tangents to the parabola can be drawn. Te shall call the points of tangency $\left(u_{0}, v_{0}\right)$, as before. Now if $X$ be the distance from the fixed point, $P$, which may as well be taken at the origin, to $\dot{u}_{0}$, and $\mathrm{Y}$ be the distance along the tangent from $u_{0}$ to $u$, it is evident that $u$ and $v w 11$ be single valued functions of $X$ and $Y$, while $X$ and $Y W 11 l$ be double valued functions of $u$ and $v$. To find the point $u_{0}$, we shall have to equate the two values of $v_{0}-$ namely, $y_{0}=m u_{0}^{2}$ and $v_{0}=a u_{0}+b$. This gives $u_{0}=\frac{m u \pm \sqrt{m^{2} u^{2}-m v}}{m}$. The steps in this solution are as 
48

follows:

The condition for equal roots, and therefore for tangency, is $a^{2}=-4 b m$, or $b=-a^{2} / 4 m$. Substituting this value of $b$ in the equation for the straight line, we have $v=a-2^{2} / 4 m$, from which $a_{0}=2 m u \pm 2 \sqrt{m^{2} u^{2}-n v}$. But $u_{0}=\frac{a}{z^{m}}$ at the point of tangency, or $u_{0}=\frac{m u \pm \sqrt{m^{2} u^{2}-m v}}{m} \quad$ Therefore $X=\int^{u_{0}} \sqrt{1+4 m^{2} u^{2}}$ and $Y=\sqrt{\left(u-u_{0}\right)^{2}+\left(v-v_{0}\right)^{2}}=\sqrt{\left(u-u_{0}\right)^{2}+\left[2 m u_{0}\left(u-u_{0}\right)\right]^{2}}=\left(u-u_{0}\right) \sqrt{1+4 m^{2} u_{0}^{2}}$ The value of $Y$ is derived by purely geometric means.

Setting up the Jacobian, we have

$$
\begin{aligned}
& \frac{\partial x}{\partial u}=\frac{\partial u_{0}}{\partial u} \sqrt{1+4 u^{2} u_{0}^{2}} \\
& \frac{\partial x}{\partial v}=\frac{\partial u_{0}}{\partial v} \sqrt{1+4 m^{2} u_{0}^{2}} \\
& \frac{\partial y}{\partial u}=\left(u-\mu_{0}\right) \frac{\psi t m^{2} \mu_{0}^{2}}{\sqrt{1+\psi m^{2} \mu_{0}^{2}}} \frac{\partial \mu_{0}}{\partial u}+\left(1-\frac{\partial u_{0}}{\partial u}\right) \sqrt{1+4 m^{2} u_{0}^{2}} \\
& \frac{\partial y}{\partial v}=\left(u-u_{0}\right) \frac{4 u^{2} u_{0}^{2}}{\sqrt{1+4 m^{2} u_{0}^{2}}} \frac{\partial u_{0}}{\partial v}-\frac{\partial u_{0}}{\partial v} \sqrt{1+42 u^{2} u_{0}^{2}} \\
& J=-\frac{1}{P^{2} \frac{\partial u_{0}}{\partial v}} \text {, where } P=\sqrt{1+4 m^{2} u_{0}^{2}} \\
& \frac{1}{v}=-\frac{1}{\frac{\partial m_{0}}{\partial v}\left(1+4 m^{2} m_{0}^{2}\right)}=\frac{-1}{\left(1+42 m^{2} u_{0}^{2}\right) \frac{1}{m}\left(I \frac{1}{2 \sqrt{2 m^{2} m^{2}-m v}}\right)} \\
& =\frac{ \pm 2 m \sqrt{n^{2} u^{2}-m v}}{1+4 m^{2} u_{0}^{2}}=0 \text {. whence } n^{2} u^{2}-m v=0
\end{aligned}
$$

or $v=$ m $^{2}$, the denied Edge of regression.

Figure 14. 


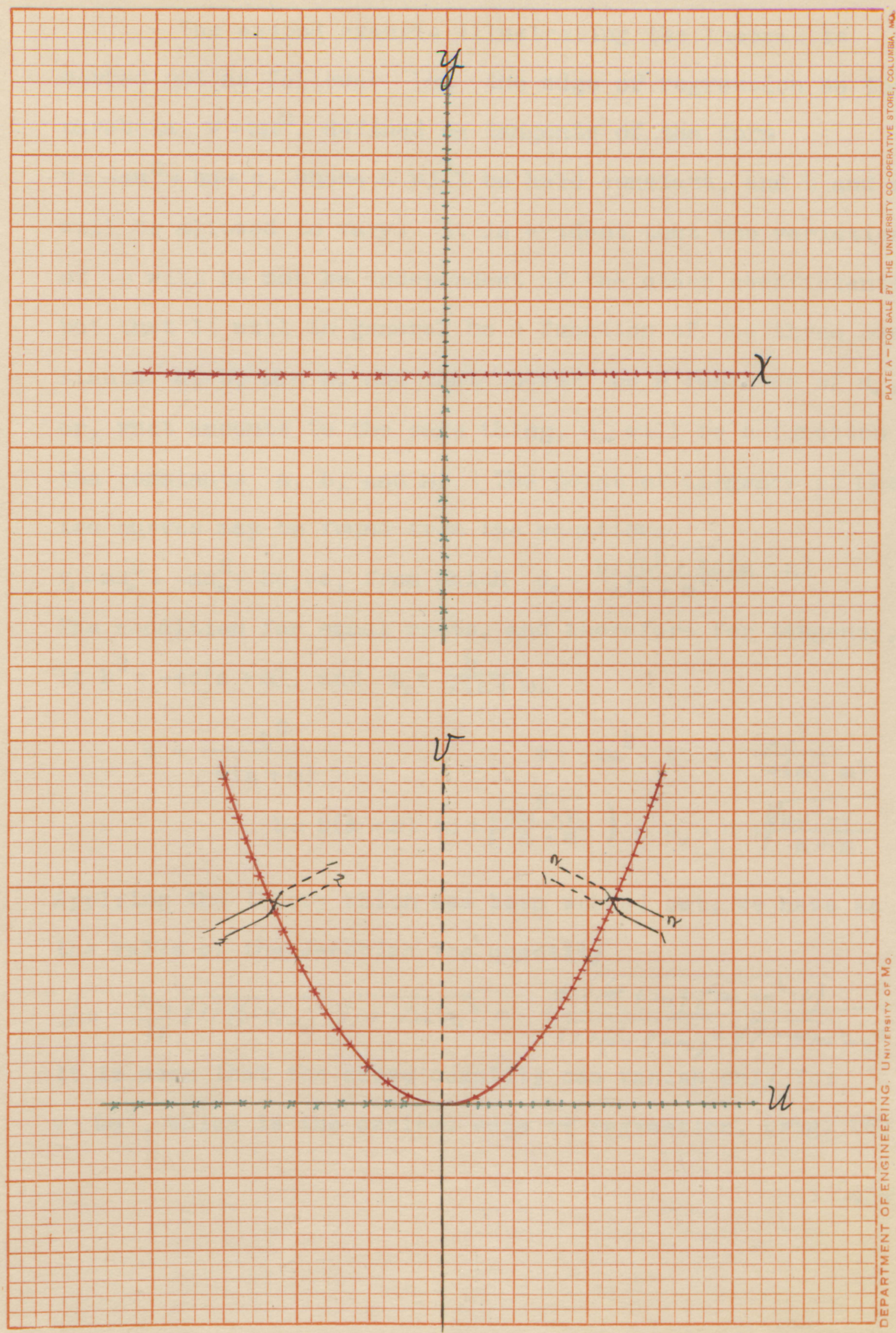


The second example illustrating the theorem is worked out as follows: let the edge of regression be the parabola $v=m u^{2}$, defined by tangents of the form $v=-a u^{2}+b u+c$, where $a w i l l$ be held constant, and $b$ and $c$ will be allowed to vary. It is evident that the variation of $c$ will be not be independent of the other coefficients, but that it will in general be a function of the three constants $a, b$, and $m$, and that $b$ will be a function of $u_{0}$, or, what amounts to the same thing, $u_{0}$ will be a function of the constants $a, b$, and $m$. The point of tangency of the two curves may be found from the equations $v_{0}=m u_{0}^{2}=-a u_{0}^{2}+b u_{0}+c$, from which $u_{0}=\frac{b \pm \sqrt{b^{2}+4 c(a+m)}}{2(a+m)}$ The condition for equal roots, and therefore for tangency, is $b^{2}=-4 c(a+m)$, and $u_{0}=\frac{b}{2(a+m)}$

$$
\begin{array}{rr}
c=\frac{-b^{2}}{4(a+m)} & v=-a u^{2}+b u+\frac{-b^{2}}{4(a m)} \\
b=2(a+m) & \left(u \pm \sqrt{\frac{m u^{2}-v}{a+m}}\right) \therefore u_{0}=u \pm \sqrt{\frac{m u^{2}-v}{a+m}}
\end{array}
$$

Applying the theory which has been rorked out, we have as the value of the Jacoblan the quantity

$$
J=-\frac{\partial u_{0}}{\partial v} \sqrt{1+\left(b-2 a u_{0}\right)^{2}} \sqrt{1+4 m^{2} u_{0}^{2}}=\frac{ \pm \sqrt{(a+i u)\left(m u^{2}-v\right)}}{2 \sqrt{1+(b-2 a u)^{2}} \sqrt{1+4 / m^{2} u_{0}^{2}}}
$$

It is seen that the above quantity can vanish only for $v=m a^{2}$, which is the desired edge of regression. (Fig, 15) 


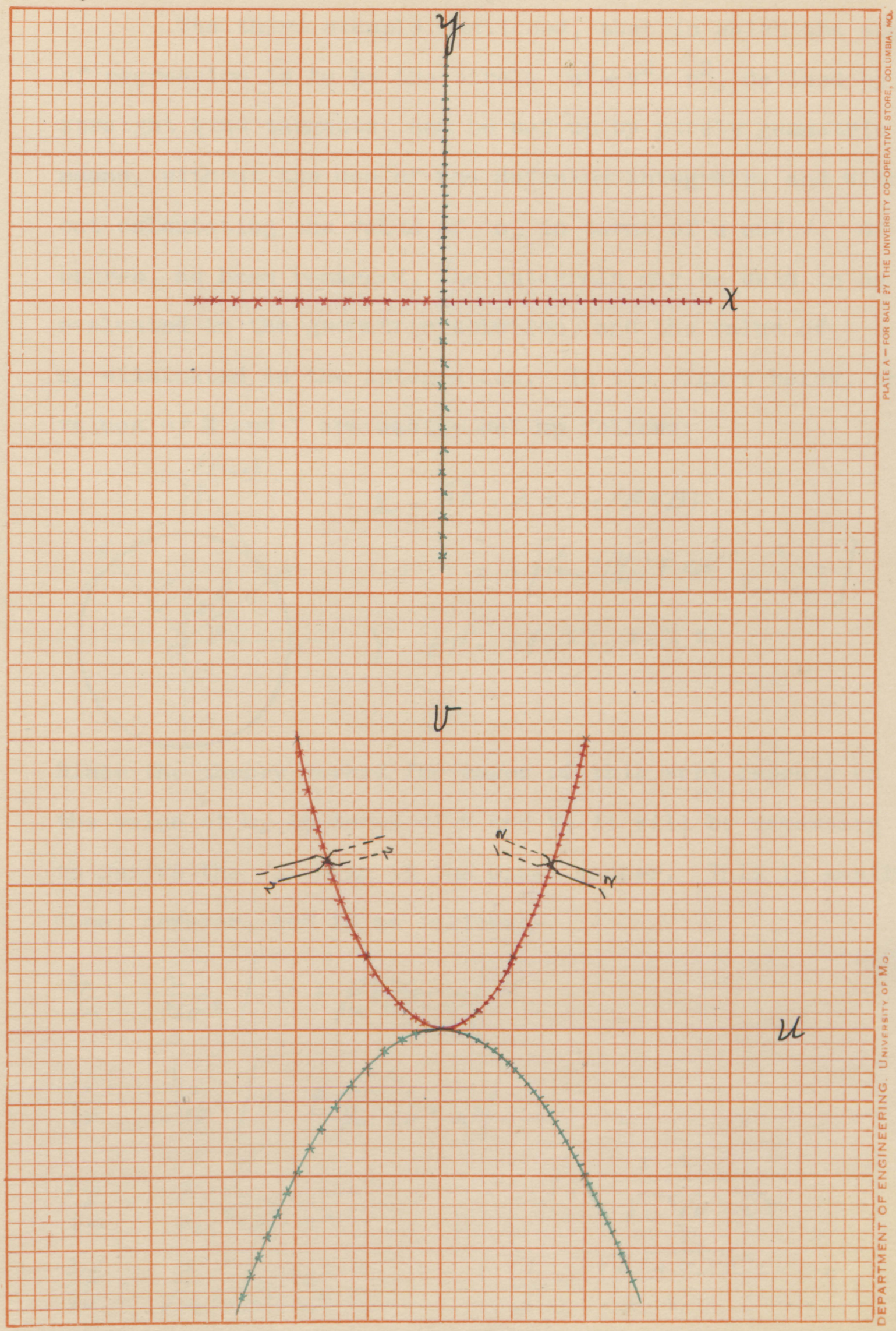


The third example mentioned, that in which the edge of regression is to be the circle $u^{2}+v^{2}=r^{2}$, defined by straight line tangents, can be set up by purely gemetric means, without the use of the definins integrals, and without calculating the value of $u_{0}$ in terms of $u$ and $v$. of course, as been remarked, the integrals may be said to be evaluated in the geometric solution, and the value of $u_{0}$ eliminated in the integration. In the figure below, the values of $\pi$ and $Y$ are easily set up follows:

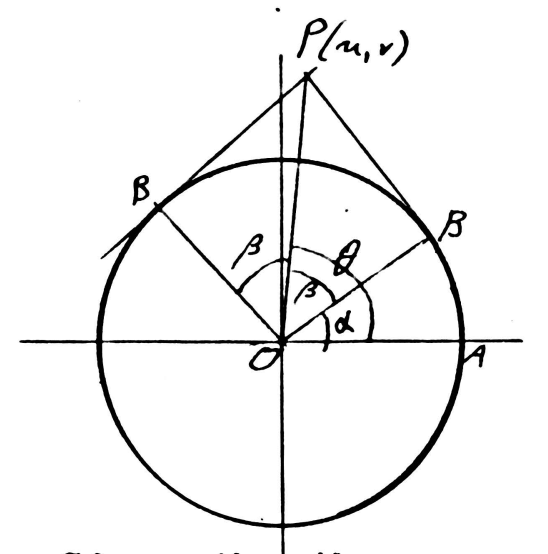

$$
\begin{aligned}
X=\widehat{A B} & =r \alpha=\Lambda(\theta \mp \beta) \\
& =\Lambda\left[\tan ^{-1}\left(\frac{v}{u}\right) \mp \cos ^{-1} \frac{r}{\sqrt{u^{2}+v^{2}}}\right] \\
Y=B P & = \pm \sqrt{u^{2}+v^{2}-\Lambda^{2}}
\end{aligned}
$$

Since the theory worked for cases of this sort depends on the value of $u_{0}$ in terms of $u$ and $v$, it will be necessary to derive the Jacobian in the usual manner. This is easily done, however, and is as follows:-

$$
\begin{aligned}
& \frac{\partial x}{\partial u}=\frac{\Omega}{u^{2}+v^{2}}\left[-v \mp \frac{u}{\sqrt{u^{2}+v^{2}-\Lambda^{2}}}\right] \\
& \frac{\partial x}{\partial v}=\frac{\Omega}{u^{2}+v^{2}}\left[u \mp \frac{v \Lambda}{\sqrt{u^{2}+v^{2}-r^{2}}}\right] \\
& \frac{\partial y}{\partial u}=\frac{ \pm u}{\sqrt{u^{2}+v^{2}-1^{2}}} \quad J=\frac{7 r}{\sqrt{u^{2}+v^{2}-r^{2}}} \\
& \frac{\partial y}{\partial v}=\frac{ \pm v}{\sqrt{u^{2}+v^{2}-r^{2}}} \quad \frac{1}{J}=\frac{\sqrt{u^{2}+v^{2}-r^{2}}}{F r}=0 \\
& u^{2}+v^{2}-u^{2}=0 \quad 2 \text { dge preg. }
\end{aligned}
$$


Since values of $X$ differing by $2 \pi r$ are congruent, it is evident that this last function is periodic, and that $2 \pi r$ is the period. The figure for the surface is given in F1E. ; but the surface is better represented on a cylinder of infinite height, with a section corresponding to the edge of regression, represented by the red line, and the part of the cylindrical surface below this section corresponding to the sheet generated by the negative end of the tangent, the part of the surface above thissction corresponding to the sheet generated by the positive end of the tangent. The line along which the surface is jolned to rake the cylindrical surface is the line marked as a cut in the two planes; hence the infinite succession of strips can be thought of as wrapped around the cylinder. The cylindrical surface can actually be formed from the UV figure by "weaving" the tangent lines until they become normal to the plane of the circle. This idea of "weaving" will be used also in the next example. (Fig. 16) 
Figure 16.
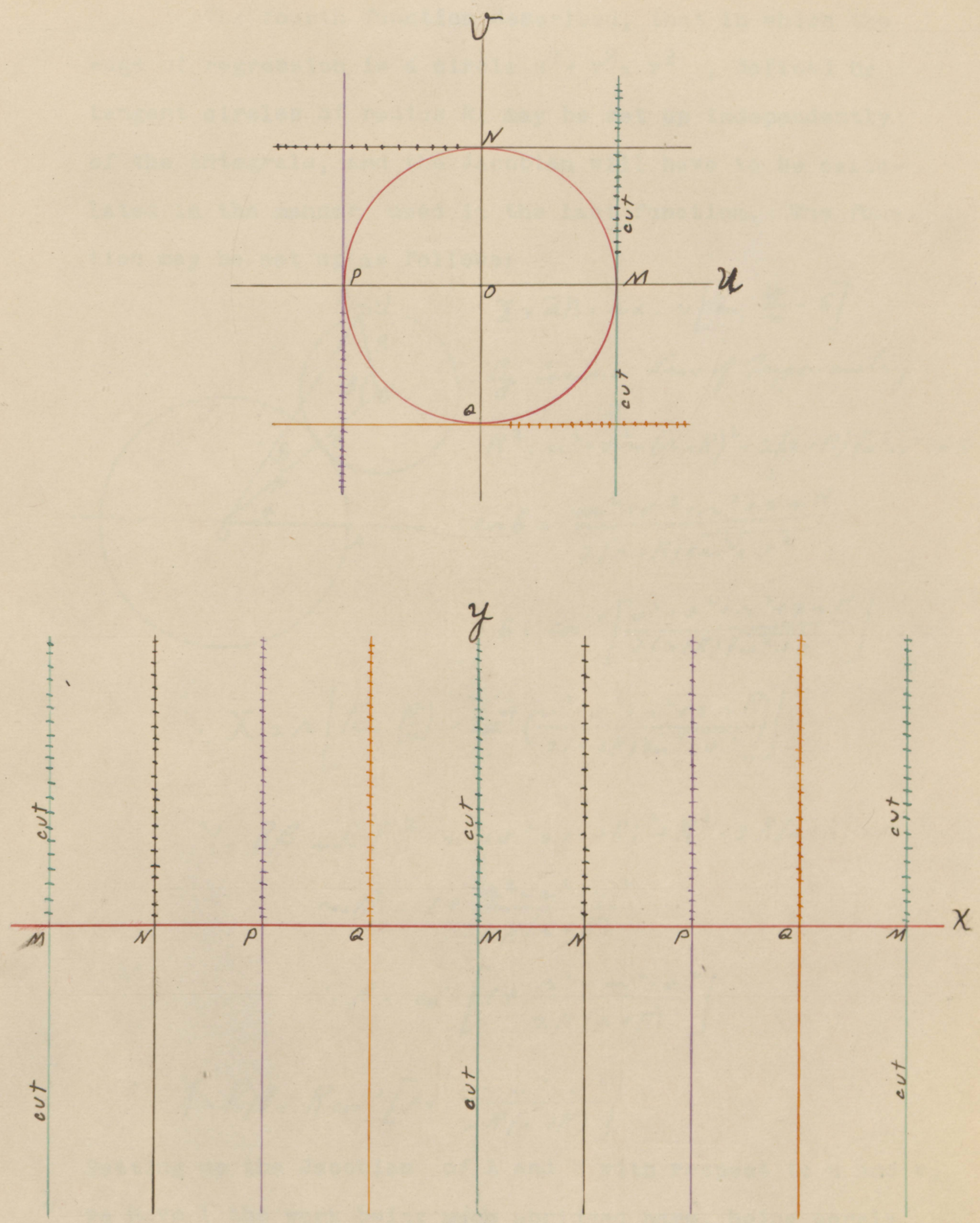
55

The fourth function described, that in which the edge of regression is a circle $u^{2}+v^{2}=r^{2}$, defined by tangent circles of radius $R$, may be set up independently of the integrals, and the Jacobian will have to be calculated in the manner used in the last function. The fundLion may be set up as follows:

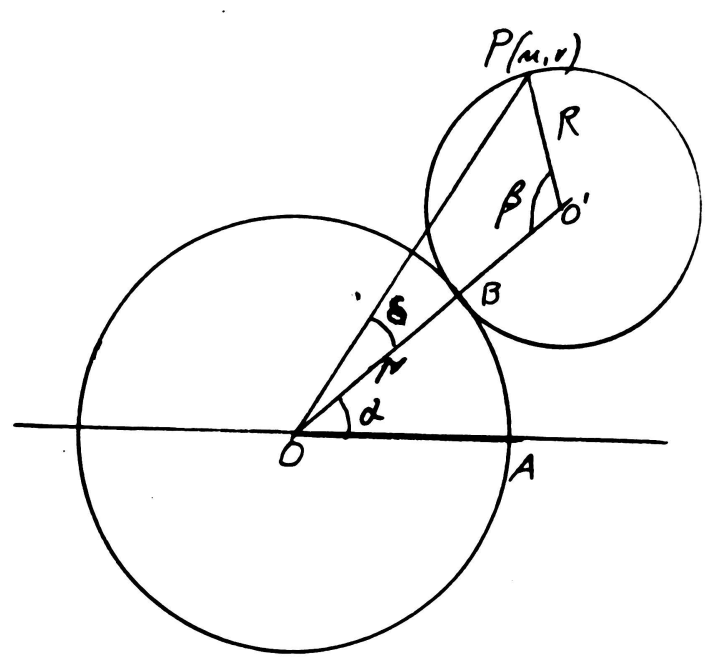

$$
X=\widetilde{a B}=\nu \alpha=\Omega\left[\tan ^{-1} \frac{\pi}{u}-\delta\right]
$$

By Canine Law fo Ingmanetry

$$
\begin{aligned}
& R^{2}=u^{2}+v^{2}+(u+R)^{2}-2(r+R) \sqrt{u^{2}+v^{2}} \cos \delta \\
& \cos \delta=\frac{u^{2}+v^{2}+\mu^{2}+2 \mu R}{2(\mu+R) \sqrt{u^{2}+v^{2}}} \\
& \delta=\cos ^{-1}\left[\frac{u^{2}+v^{2}+r^{2}+2 \mu R}{2(u+R) \sqrt{u^{2}+v^{2}}}\right]
\end{aligned}
$$

$$
\begin{aligned}
& \therefore X=r\left[\tan ^{-1}\left(\frac{\mu}{u}\right)-\cos ^{-1}\left(\frac{\mu^{2}+v^{2}+\mu^{2}+2 \mu R}{2(\mu+R) \sqrt{u}^{2}+v^{2}}\right)\right] \\
& Y=R \beta \text { and } \overline{O P}^{2}=\mu^{2}+v^{2}=(R+R)^{2}+R^{2}-2 R(\alpha+R) \cos \beta \\
& \cos \beta=1+\frac{u^{2}-u^{2}-v^{2}}{2 R(\mu+P)} \\
& \therefore \beta=c_{\infty}^{-1}\left[1+\frac{2^{2}-\mu^{2}-v^{2}}{2 R(n+R)}\right] \\
& Y=R P=\operatorname{Per}^{-1}\left[1+\frac{1^{2}-\mu^{2}-v^{2}}{2 R(n+R)}\right]
\end{aligned}
$$

Setting up the Jacobian of $X$ and $Y$ with respect to $u$ and $v$, we have ( the work being much abridged here, being merely formal differentiation) 
56

$$
\begin{aligned}
& \frac{\partial x}{\partial u}=r\left[\frac{-v}{u^{2}+v^{2}} \mp \frac{u\left(u^{2}+v^{2}-2 r R-r^{2}\right)}{\left(u^{2}+v^{2}\right) \sqrt{f(r+R)^{2}\left(u^{2}+v^{2}\right)-\left(u^{2}+v^{2}+r^{2}+2 r R\right)^{2}}}\right]
\end{aligned}
$$

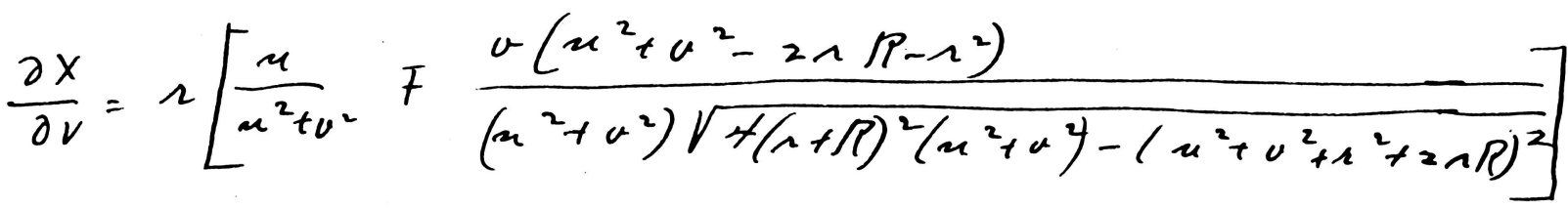

$$
\begin{aligned}
& \frac{\partial y}{\partial u}=\frac{2 R_{u}}{\sqrt{H P^{2}(\Lambda+P)^{2}-\left[2 R(n+P)-\left(u^{2}+v^{2}-r^{2}\right)\right]^{2}}} \\
& \frac{\partial Y}{\partial v}=\frac{2 p v}{\sqrt{4 R^{2}(1+P)^{2}-\left[2 R(n+P)-\left(u^{2}+v^{2}-1 n^{2}\right)\right]^{2}}} \\
& \therefore J=\frac{-2 \pi n\left(u^{2}+v^{2}\right)}{\sqrt{4 R^{2}(n+R)^{2}-\left[2 R(u+R)-\left(u^{2}+v^{2}-n^{2}\right)\right]^{2}}} \\
& \frac{1}{J}=0=\sqrt{4 p^{2}(n+R)^{2}-\left[2 R(2+R)-\left(u^{2}+v^{2}-n^{2}\right)\right]^{2}} \text {, sizes the }
\end{aligned}
$$

denominator of canst wancich.

$$
\begin{aligned}
\therefore 2 R(1+R)-\left(u^{2}+v^{2}-r^{2}\right) & = \pm 2 R(n+R) \\
u^{2}+v^{2}-r^{2} & =72 R(n+R)+2 R(n+R)=0 u^{4}+R(n+R) \\
u^{2}+v^{2} & =r^{2} a r^{2}+4 P r+4 R^{2}=(n+2 R)^{2}
\end{aligned}
$$

The function originally set up has therefore two edges of regression, namely. $u^{2}+v^{2}=r^{2}$ and $u^{2}+v^{2}=(r+2 R)^{2}$, which is what might be expected, since the circle defined by the 
envelope of the circle of radius $R$ is also an edge of regression. Since $X$ and $Y$ are each expressed as inverse trigonometric functions of angles, it is evident that values of $X$ and $Y$ differing by $2 \pi r$ and $2 \pi R$ respectively will be congruent, and the function $(X, Y)$ is therefore doubly periodic, with periods $2 \pi r$ and $2 \pi R$ respectively. (Fig.17)

It is evident from the above that we can represent this surface on the curface of an anchor ring. Referring to the figure, annular ring bounded by the two red circles, and indicated in section by the green circles, can be distorted, by the "weaving" process mentioned before, to show such a surface as the anchor ring. The same surface can be secured by taking one of the rectangles bounded by red lines, at the bottom of figure 17, and folding first along the clear red line until the marked red lines coincide, and then bringing the tube thus formed end to end, so the marked green lines coincide. The two periods are then the circumference of the marked red circle and the circumference of the marked green circle, and the whole surface consists of an infinite number of sheets on the anchor ring, but periodic as indicated. 

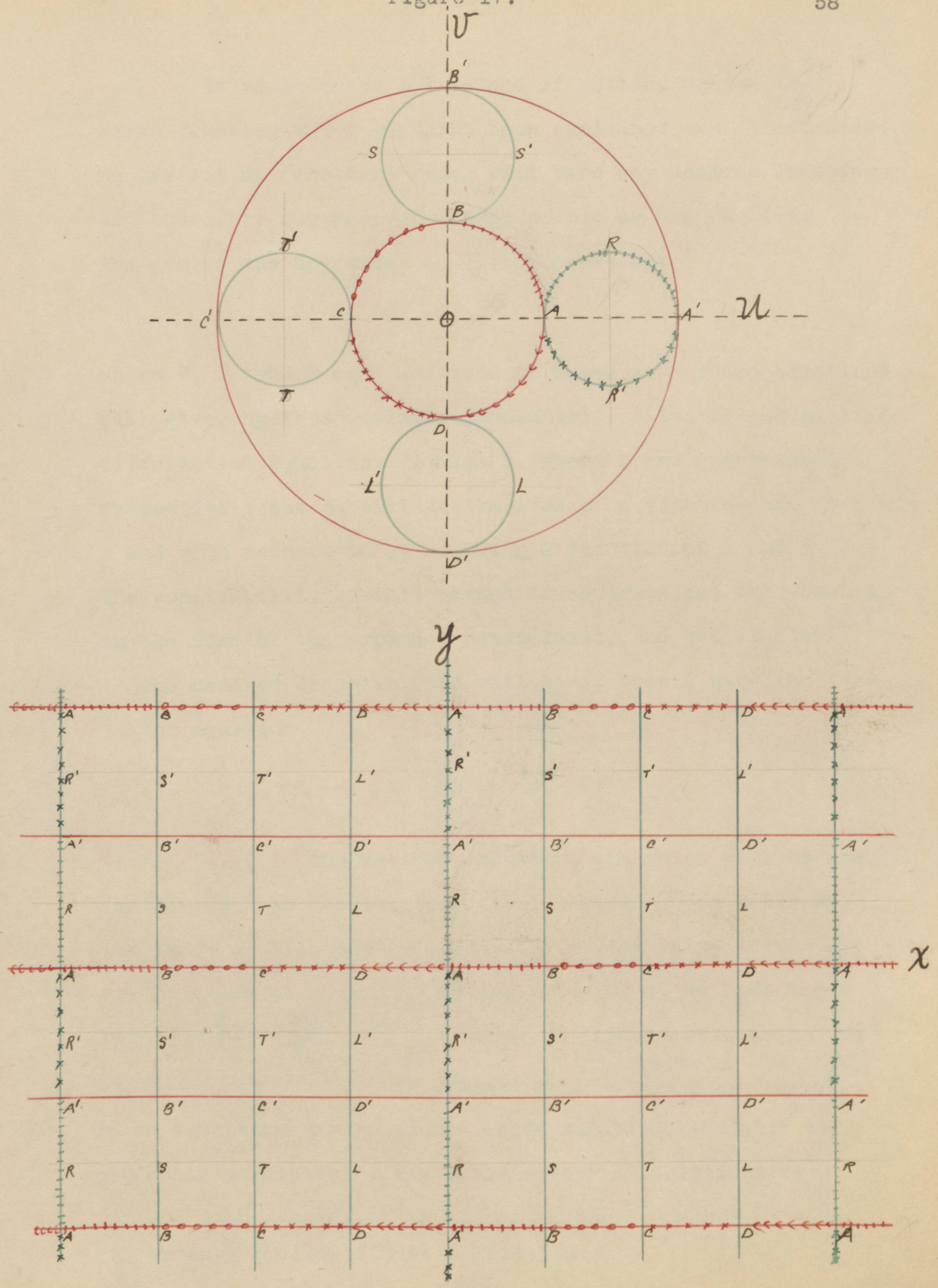
In addition to the method of setting up the desired function which has just been explained and illustrated, we may set up functions which will have any desired Jacobian, and therefore any desired branch points or branch lines, by the use of the Lagrange auxiliary equations 1

$$
\frac{d x}{p}=\frac{d y}{Q}=\frac{d z}{R}
$$

where $P, Q$, and $R$ are functions of $x$ and $J \cdot$ These equations will give, by their solution, solutions of the linear partial differential equation $P p+Q q=R$, where $p$ and $q$ represent respectively the partial derivatives of $z$ with respect to $x$ and with respect to $y, z$ being $a$ function of $x$ and $y$. The applicability of this method if we write out the Jacobian in the form of tho expanded determinant, and set it equal to the desired Jacobian. Me will have, then, have the following equation

$$
\frac{\partial u}{\partial x} \frac{\partial v}{\partial y}-\frac{\partial u}{\partial y} \frac{\partial v}{\partial x}=F(x, y)
$$

where $F(x, y)$ is the desired Jacobian, and which will be used instead of $R$ in the bove solution. Now if $\frac{\partial v}{\partial y}$ be taken as the value of $\boldsymbol{p}$ in the solution, $\frac{\partial u}{\partial x}$ as the value of $p,-\frac{\partial v}{\partial x}$ as the value of $Q$, and $\frac{\partial x}{\partial y}$ as the value of $q$, we have the equation $\frac{d_{x}}{\frac{\partial v}{\partial y}}=\frac{d y}{-\frac{\partial v}{\partial x}}=\frac{d u y}{F(x, y)}$, using $u$ instead of $z$ as in the original solution. It is assumed that $v$ has been chosen in an arbitary manner in the above solution, and this is perfectly permissible, since the number of solutions is 
Infinite. Now the solution of the Lagrange equations will give as one solution $v=f(x, y)=K$, from which a value of $y$ in terms of $x$ and the constant $K$ can be determined. If this value of $y$ be then substituted in $F(x, y)$, and the equation $\frac{d x}{P}=\frac{d u}{F(x, y)}$ be solved for $u$, which will be easily carried out, the value of $u$ thus derived, together with the value of $v$ originally chosen, will be one of the required functions which w1ll produce the desired Jacobian. It is evident that the cholce of the expression for $v$ will in a large measure determine the difficulty of the problem; for if an expression be chosen which is difficult to solve for $y$ in terms of $x$ and the constant $K$, the problem becomes in general difficult, though in some cases a complicated expression for $v$ may give a simple value for $u$. Some examples may be chosen illustrating the above theorem, as, for instance, the problem to discdver an expression for $u$ which, together with a chosen value of $v$, as $x y$, will give the Jacobian $x^{2}-y=0$. This example is chosen because it was one of the examples discussed in the last chapter. If this value of $v$ be set equal to $K$, we have $\mathrm{y}=\mathrm{K} / \mathrm{x}$. Then we have the equation $\frac{\mathrm{dx}}{\mathrm{x}}=\frac{\mathrm{du}}{\mathrm{x}^{2}-K / \mathrm{x}}$ to solve. The solution of this equation is $x^{2} / 2+K / x=u$, which, after substituting for $K$ its equal $x y$, gives $u=x^{2} / 2+y$. The example referred to gave as the Jacobian $2\left(x^{2}-y\right)$; hence if the value of $u$ derived above is multiplied by two, we will have the value of $u$ which was used in the problem in the earlier chapter. 
In applying the Lagrange method, special devices are sometimes advantageous in solving the equations. An example which ras studied in the preparation of this paper, though the surface is not drawn here, is a function which will give the Jacobian $x^{2}+y^{2}-4$, which would give the circle indicated as the branch Iine. In this case, $V$ was chosen as $y(x)$ 2). Then we have to solve the equations $\frac{d x}{x+2}=\frac{d y}{-y}=\frac{d u}{x^{2}+y^{2}-4}$. Now if the first fraction be multiplied in both terms by $(x-2)$, the second fraction by $y$, and then the principle of proportion by composition be applied, we have $\frac{(x-2) d x+y d y}{x^{2}+y^{2}-4}=\frac{d u}{x^{2}+y^{2}-4}$ from which $u=\frac{x^{2}}{2}-6 x+\frac{y^{2}}{2}$. This value of $u$ can be multiplied by a constant without affecting the Jacobian other than by a constant factor; hence we may take $x^{2}-4 x+y^{2}$. for the value of $U$, with the chosen value of $V$ - that is, $V=y(x+2)$. Other special devices will be useful in individual problems, bit no attempt need be made here to discuss these devices. 


\section{BIBL IOGRA PHY}

Goursat, Edouard, A Course in Mathematical Analysis. Translated by E. R. Hedrick. Ed. 1, Valume I, Boston, Ginn and Company, 1904.

Hurray, David A. Introductory Course in Differential Equations, Ed. 12, New York, Longmans, Green, and Co., 1914. 


\section{$378.7 M 71$ \\ $\times J 64$}

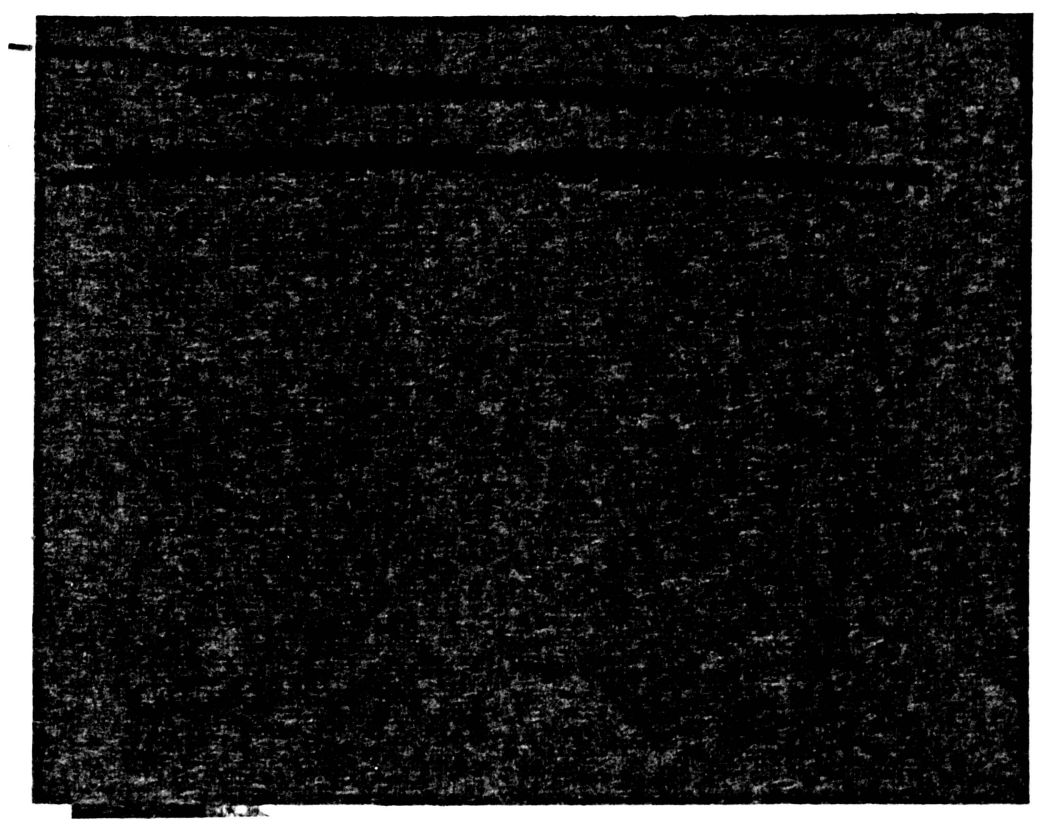


